\title{
Studying the evolution of social behaviour in one of Darwin's Dreamponds: a case for the Lamprologine shell- dwelling cichlids
}

\author{
Etienne Lein $(\mathbb{D} \cdot \operatorname{Alex}$ Jordan $\mathbb{D}$
}

Received: 17 April 2020/Revised: 22 October 2020/Accepted: 18 November 2020/Published online: 24 February 2021

(C) The Author(s) 2021

\begin{abstract}
The link between the evolution of advanced sociality and cognition has been an important concept across fields and taxonomic boundaries. However, in many study systems, ecological and phylogenetic confounds impair evolutionary inferences drawn when comparing social organization. Here, we highlight the value of the shell-dwelling Lamprologine cichlids of Lake Tanganyika in studies of cognitive and social evolution. These species show differences in social organization, both within and
\end{abstract}

\begin{abstract}
The term "Darwin's Dreampond" was coined in the eponymous popular book by Tijs Goldschmidt (1996) to describe and celebrate the adaptive radiation of Lake Victorian cichlids. Goldschmidt compared this radiation to Galapagos Finches and Hawaiian Honeycreepers, and it was argued that, had Darwin known about the diversity in Lake Victoria, it would have been among his dream systems to explore his ideas of evolution and speciation. Yet for studies of behaviour and sociality, we believe it is another of the Great Rift Lakes, Lake Tanganyika that can be considered a Darwinian "Dreampond" - the long evolutionary history, incredible diversity of behaviour, and complex social organization of Tanganyikan cichlids provides the ideal system in which to study processes of behavioural evolution and diversification.
\end{abstract}

Guest editors: S. Koblmüller, R. C. Albertson, M. J. Genner, K. M. Sefc \& T. Takahashi / Advances in Cichlid Research IV: Behavior, Ecology and Evolutionary Biology

E. Lein $(\bowtie) \cdot$ A. Jordan

Department of Collective Behaviour, Max Planck Institute of Animal Behavior, Konstanz, Germany

e-mail: leinetienne@gmail.com across species, but otherwise exhibit remarkable similarities in their ecology and life history. We focus on the ecological and social attributes of 15 Lamprologine cichlids that live in permanent association with empty gastropod shells, often in syntopy and with largely overlapping ecological niches. We then discuss difficulties with terminology and categorization of social organization, outlining current and emerging methodologies to address these limitations. Our goal is twofold: (i) to gather available empirical evidence on the behaviour, life history, and ecology of shelldwelling Lamprologine cichlids, highlighting their potential in comparative studies of cognition and evolution, and (ii) to stimulate debate and critical appraisal of current terminology and categorizations of social structure, ideally leading to more precise and empirically standardized definitions of sociality in cichlids.

Keywords Cichlid - Social - Evolution - Brain · Cognition $\cdot$ Lake Tanganyika

\footnotetext{
E. Lein · A. Jordan

Centre for the Advanced Study of Collective Behaviour, University of Konstanz, Konstanz, Germany
} 


\section{Introduction}

Social interactions are ubiquitous in the animal world, from territorial behaviour in otherwise solitary animals, to brief mating unions, through to permanent social bonds; the consequences of social interactions are complex and far reaching. When considering the evolution of behaviour, the role of the social environment presents a fascinating and challenging suite of conceptual, theoretical, and empirical problems. At a broad level, the fitness consequences of living socially are well understood (Wilson, 2000; Rubenstein \& Abbot, 2017). Alexander (1974) was the first to note that group living will only evolve when there are sufficient benefits to offset the costs of increased disease and parasite transmission and intensified competition, and these potential costs have been well studied in a variety of taxa (Booth, 1995; Slotow \& Paxinos, 1997; Martinez \& Marschall, 1999; Coolen, 2002; Krause et al., 2002). The benefits of group living are also well documented (Krause et al., 2002) and generally include increased foraging success (Krebs, 1974; Pitcher et al., 1982; Morgan, 1988), and reduced per-capita predation risk, encompassing increased predator vigilance (Morgan, 1988; Lima, 1995; Lima \& Bednekoff, 1999), predator confusion (Pitcher et al., 1982; Grand and Dill 1999), and predation dilution (Pitcher, 1986; Krause et al., 2002). While these general costs and benefits are relatively well understood, understanding the selective pressure exerted by the social environment on individual level behavioural phenotypes remains difficult. Perhaps the most difficult element of this problem is that social environments are fluid, and the high spatial and temporal variability of exchanges with social partners makes it difficult to understand how these interactions result in selection on behaviour. Moreover, rather than being passive subjects of selection induced by social context, individuals can change the selective environment they experience by moving between social groups, potentially entering contexts that are more favourable in terms of, e.g. sex ratio, reproductive opportunities, or decreased competition (Oh \& Badyaev, 2010; Jordan $\&$ Brooks, 2012). The fluid nature of social contexts further complicates any analysis of how social context shapes the evolution of behaviour.

Despite the challenges, examinations of the link between the degree of "social complexity" (a metric of how many interactions an individual may experience, and how varied these interactions might be), and an individual's degree of "behavioural complexity" (a range of behaviours an individual can perform), has been a fruitful ground for conceptual development. From early analytical models of the game-theoretic nature of animal behaviour (Grafen \& Sibly, 1978; Smith, 1977), in which the value of any particular strategy should be considered in light of its performance against competing strategies, to empirical tests of the value of different behavioural strategies in varying social regimes (Jordan et al., 2014; Parker, 1974), up to broad conceptual syntheses of the value of social context-appropriate responses (Taborsky \& Oliveira, 2012) - the interaction between social context and individual behaviour has long fascinated researchers. Major questions in the field of social behaviour are still wide open. Why do some species engage more frequently in these social interactions than others? Which factors determine whether an animal lives solitary, in aggregations, or in stable social groups? Although being challenging questions to answer, uncovering the proximate and ultimate factors shaping sociality are essential steps towards an understanding of the evolution of social behaviour. In this paper, we will argue that shell-dwelling cichlids of Lake Tanganyika represent a powerful study system that will yield answers to many of these seemingly insurmountable challenges.

\section{Social evolution and its consequences for behaviour and the mechanisms of behaviour}

While living in groups is advantageous for many reasons, there are clearly limitations on the major evolutionary transition to sociality (Szathmáry \& Smith, 1995). Social behaviour is poorly represented in many taxa. For instance, of the $\sim 48,000$ accepted species of spiders (www.wsc.nmbe.ch, 2019), fewer than 100 are considered social by certain standards (Lubin \& Bilde, 2007). This is likely due to the inherent conflict associated with living in groups of potential competitors (Davies et al., 2012), providing internal threat to these temporally stable "communities of fate". The question then arises as to how individuals manage to navigate such complex social arrangements. The concept of 'social competence' (Taborsky \& Oliveira, 2012; Varela et al., 2020) has sought to explain the link between the demands of 
social interactions and the evolution of advanced socially relevant cognitive skills. Social competence is defined as the ability of an animal to adaptively adjust the expression of its social behaviour according to previous social experience and social context, using a number of building blocks including cognitive appraisal, social recognition and memory, and social inference (Oliveira, 2013). For example, in Neolamprologus pulcher, a Lamprologine cichlid species that forms stable social groups, individuals that had been reared in socially more rich environments show more submissive and less aggressive behaviours in a contest with a more dominant conspecific and thus decrease the probability of being evicted from the territory (Fischer et al., 2015; Nyman et al., 2017).

Associated with this increased cognitive complexity is the development of mechanisms that produce social behaviour. In addition to the various genetic, endocrinological, and other physiological processes underlying social behaviour, it is neuroanatomy, and patterns of neural activity that have received most research attention. While the general concept has been formulated before in many variations, the best known is the Social Brain Hypothesis (SBH; Dunbar, 1998; Humphrey, 1976; Whiten \& Byrne, 1988). This hypothesis argues that sociality poses increased cognitive challenges that lead to the evolution of individuals with more complex cognitive and neuroanatomical traits (Jolly, 1966; Whiten \& Byrne, 1988). When first published, the hypothesis aimed to test the idea that primates living in larger social groups showed disproportionately large brains (usually indexed as neocortex volume) relative to their body size (Dunbar, 1992; Dunbar, 1998; Whiten \& Byrne, 1988). Arguing that evolution typically constitutes an economical process that balances costs and benefits, the high operating expenses, and surplus of brain tissue (beyond the body's pure physical requirements) were suggested to be compensated for by enhanced cognitive skills. These increased cognitive skills in turn allowed individuals to cope with the challenges related to a complex social life style and accrue benefits of group living; this hypothesis, therefore, proposed a direct evolutionary relationship between sociality, cognition, and brain size. In the following years, the SBH was expanded to attain a wider evolutionary application by extending the concept also to other group living, non-primate taxa, such as ungulates, carnivores, bats, birds, and even insects - however, with varying and inconclusive results (Dunbar \& Shultz, 2007; Lihoreau et al., 2012).

\section{Disentangling complexity in social and ecological dimensions}

In order to investigate the putative link between sociality and cognitive capacity, it is essential to eliminate equally plausible alternative theories that have been brought forward as explanations for observed variations in brain size or cognitive capacity more generally (Dunbar \& Shultz, 2007). In the case of primates, brain size has been associated for instance with the way in which food is processed ("extractive foraging hypothesis"; Gibson, 1986; Parker \& Gibson, 1977), with dietary preferences (higher unpredictability of fruits as compared to leaves; Clutton-Brock \& Harvey, 1980; DeCasien et al., 2017), or with differences in home ranges (larger ranges potentially require more sophisticated mental maps; Clutton-Brock \& Harvey, 1980). At the same time, the SBH has been criticized for inaccuracy related to the (initial) concept of "social complexity" (Dunbar \& Shultz, 2007; Healy \& Rowe, 2007; Logan et al., 2018).

In a formal analysis of the evolution of behaviour, cognition, and neuroanatomy, it is necessary to separate social from other ecological factors (Barton, 1996; Dunbar \& Shultz, 2017; Reader \& Laland, 2002; Shumway, 2010). In a previous study investigating seven species of the cichlid fish tribe Ectodini, habitat complexity, and measures of social organization were found to co-vary, confounding a resolution of the sources of selection driving changes in brain size (Pollen et al., 2007). In order to address these issues, a common strategy is to explicitly incorporate all recorded ecological variables into a single statistical analysis (Shultz \& Dunbar, 2006; DeCasien et al., 2017). An example for this approach is the use of multiple regression analysis. However, in studies with small sample sizes and strong multicollinearity between variables, this kind of statistical analysis can regularly prevent a clear separation, and thus interpretation, of predictors (Deaner et al., 2000; Gonzalez-Voyer et al., 2009). However, even with the most robust statistical tools, the interaction amongst social and other ecological factors remains a conceptual problem in understanding the sources of selection acting on sociality. By contrast, a study system 
exhibiting minimal variation with respect to all relevant ecological variables, but high variation in the variable of interest-in this case social complexity-would be ideal for testing predictions made by the SBH (Gingins \& Bshary, 2016), namely that increased social complexity drives the evolution of cognitive capacity. Here, we make the argument that the shell-dwelling Lamprologine cichlids of Lake Tanganyika offer just such a system for understanding the co-evolution of social structure, behaviour, cognition, and neuroanatomy.

\section{Cichlid fish as model systems}

Compared to taxa such as primates (e.g. Reader \& Laland, 2002; DeCasien et al., 2017) or birds (e.g. Beauchamp \& Fernández-Juricic, 2004; Kabadayi et al., 2016; Shultz \& Dunbar, 2010), fish have traditionally played a comparatively minor role in the study of cognitive evolution (Bshary et al., 2014). Nevertheless, the utility of fishes as a model system has been recognized by a handful of fairly recent multi-species comparisons (Pollen et al., 2007; Chojnacka et al., 2015; Soares et al., 2015; Gingins \& Bshary, 2016; Reddon et al., 2017), or studies investigating intraspecific variation in the social environment (Arnold and Taborsky 2010; Fischer et al., 2015; Kotrschal et al., 2012; Taborsky et al., 2012; Wismer et al., 2014; Triki et al., 2019). Given their impressive diversity in terms of social organization and the exploitation of different ecological niches (Fryer \& Iles, 1972; Meyer, 1993), it is not surprising that Tanganyikan cichlids have been the focus in the majority of these studies. In a study of seven species of the Tanganyika cichlid tribe Ectodini, social and physical environment were associated with the volume of major brain regions. Specifically, telencephalic size was found to be larger in monogamous as compared to polygamous species; at the same time, however, habitat complexity (measured as rugosity) showed a similar trend (Pollen et al., 2007). A comparative study including 39 Tanganyikan cichlid species from six different tribes found diet type (whose rank was based on increasing prey motility) to be negatively correlated with brain weight (Gonzalez-Voyer et al., 2009), a result that was interpreted to be linked to the more complex social structure associated with diet via habitat (although habitat complexity itself was not directly correlated with brain size; Gonzalez-Voyer et al., 2009).

A subsequent study by the same authors investigated 43 Tanganyikan cichlid species and found mating competition (which correlates with mating system type; Gonzalez-Voyer et al., 2008) to be correlated negatively with telencephalon volume, whereas habitat complexity (measured as rugosity) was found to be positively correlated with telencephalon volume (Gonzalez-Voyer and Kolm, 2010), thus, confirming previous results (Pollen et al., 2007). By contrast, the study found no association between diet type and telencephalon size (Gonzalez-Voyer \& Kolm, 2010) as had been predicted by the authors beforehand on the basis of prior results (GonzalezVoyer et al., 2009). This exemplifies well the complex effects and interactions that different environmental variables such as habitat (e.g. rugosity, depth) or diet (e.g. feeding type) on the one hand (Huber et al., 1997; Sylvester et al., 2010), and social variables (e.g. mating system type, parental care) on the other hand (Pollen et al., 2007; Shumway 2008; Gonzalez-Voyer et al., 2009; Gonzalez-Voyer and Kolm 2010), exert on brain size evolution in Tanganyikan cichlids (and analogously on other vertebrate study systems, like carnivores or primates; Gittleman 1986; Dunbar and Shultz 2017).

The interpretation of the results in these and similar studies is made difficult when study animals are sourced from commercial collectors. Sourcing animals from exporters risks creating confusion over collection location, ontogeny, and rearing conditions of "wild-caught" animals (which may have spent some generations in collector's and exporter's ponds outside the lake itself; AJ personal obs.), and even confusion over the species assignment itself, given that closely related species may have overlapping distributions separated only by local differences in habitat. As an example of this latter point, Telmatochromis temporalis and Telmatochromis temporalis 'dwarf morph' occur in close proximity but occupy different ecological niches and have divergent life histories (Takahashi, 2004; Takahashi \& Ota, 2016). Despite clear variation in social behaviour and organization, both these variants are described as $T$. temporalis in the aquarium trade and in much of the scientific literature. If an understanding of ecological factors mediating evolution of a trait is sought, it is necessary to have an accurate estimate of those 
ecological factors, especially considering that shortdistance changes in conditions can create large differences in the selective environment experienced by an individual (Bolnick et al., 2007; Richardson et al., 2014; Maciejewski et al., 2020). Local environmental conditions vary substantially in Lake Tanganyika, and such small-scale variation has been shown to affect selection regimes in other aquatic environments (Maciejewski et al., 2020). Because of the lack of detail on actually experienced selective environments, it is challenging to draw conclusions about the interaction among ecological conditions and the evolutionary process when assuming species-level attributes.

Instead of approaches that seek to explicitly account for a number of confounding variables, an alternative strategy is to identify a set of species in which these confounds are minimized (Shumway, 2008). We argue that the Lamprologine shell-dwelling cichlids of Lake Tanganyika are one of the best examples of such a system. The shell-dwelling cichlids are a species group that possess many valuable attributes in studies of social evolution and behaviour: (i) at 15-23 species, a powerful comparative sample (Table 1), (ii) with close and wellresolved phylogenetic relationships, (iii) and similar body sizes; similar ecological factors, including (iv) overlapping dietary niches with similar feeding modalities, and (v) sympatric patterns of distribution, or even syntopic mosaic communities with similar risk environments on the macro-habitat level, and (vi) a low variation in environmental complexity, due to their resembling, permanent, association with empty snail shells; (as a result of this shell-dwelling lifestyle, it is conceivable that also other life-history traits, including brood size or age at sexual maturity, are similar across these cichlid species). Crucially, despite these remarkable similarities, the shell-dwelling Lamprologine cichlids are highly divergent with regard to their social structure. It is this combination of attributes that makes shell-dwelling cichlids so wellsuited for investigations into the influence of the social environment on cognitive and behavioural evolution. In the following section, we explain in detail each of these attributes across the shell-dwelling cichlid species.

\section{Attributes of Lake Tanganyikan shell-dwelling cichlids}

One of the Great Lakes in the East African Rift Valley, Lake Tanganyika is shared between the countries of Tanzania, Zambia, D.R. Congo, and Burundi. The world's second largest body of freshwater and home to a multitude of endemic species, Lake Tanganyika is famed for its astonishing diversity of cichlid fishes (currently 208 valid species; estimates reach up to 241 species; Ronco et al., 2019), which, all but a few exceptions, inhabit its near-shore benthic zone (Konings, 2015).

\section{Phylogeny}

A model system for ecology and evolution (Salzburger et al., 2005) and textbook example in evolutionary biology for rapid and extensive adaptive radiation (Kocher, 2004; Seehausen, 2006; Takahashi and Koblmüller 2011), Lake Tanganyikan cichlids have been subject to intense scientific interest spanning a period of over 120 years (R.T. Günther described the first cichlid species in 1894, G.A. Boulenger in 1897). Among other important achievements, this sustained research has recently yielded one of the best resolved molecular phylogenies for any radiation to date (Ronco et al., accepted). This detailed knowledge of the phylogenetic relationships of Tanganyikan cichlids generally, and Lamprologine (Sturmbauer et al., 2010) or shell-dwelling cichlids (Koblmüller et al., 2007) specifically, enables a powerful use of the comparative method and is a fundamental prerequisite for testing evolutionary hypotheses (MacLean et al., 2012). The fact that Tanganyikan cichlids evolved more rapidly as compared to other vertebrate lineages potentially makes it easier to identify salient selection pressures (Pollen et al., 2007). Especially in closely related species, in which variability is expected to be limited by similar developmental constraints, discovering such variability for a trait across closely related species might be evidence that this trait has been shaped by selection pressure (Pollen et al., 2007).

While the species flock of Tanganyikan cichlids comprises sixteen different tribes, it has been the monophyletic tribe of Lamprologine cichlids that, as a consequence of their immense diversity in social organization and sophistication of social behaviours 
Table 1 Syntopies, body lengths, and modality of shell use in permanently shell-dwelling cichlids; light red shaded cells denote further potential candidate species for shell-dwelling, albeit with currently sparse information; SL: standard length, TL: total length

\begin{tabular}{|c|c|c|c|c|c|}
\hline \multirow{2}{*}{ Species } & \multirow{2}{*}{ Confirmed syntopy [species] } & \multirow{2}{*}{ Length [cm SL | TL] } & \multicolumn{2}{|c|}{ Shell use } & \multirow{2}{*}{ References } \\
\hline & & & brooding & shelter & \\
\hline $\begin{array}{l}\text { Neolamprologus multifasciatus } \\
\text { BOULENGER } 1906\end{array}$ & 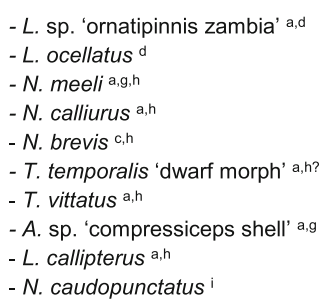 & $\begin{array}{l}1.90-2.45 \mathrm{~cm} \mathrm{SL} \\
\text { (median) }^{d} \\
2.5-4 \mathrm{~cm} \mathrm{TL}^{\mathrm{e}}\end{array}$ & $\checkmark 1, a-f$ & $++1, a-f$ & 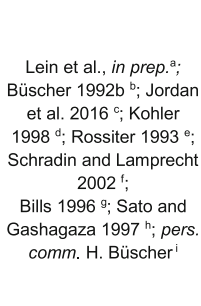 \\
\hline $\begin{array}{l}\text { Neolamprologus similis } \\
\text { BÜSCHER } 1992\end{array}$ & $\begin{array}{l}\text { - L. speciosus }{ }^{2, a, b} \\
\text { - L. callipterus }{ }^{d} \\
\text { - N. caudopunctatus }{ }^{d}\end{array}$ & $\begin{array}{l}2.66-3.58 \mathrm{~cm} \mathrm{SL} \\
\text { (range of sizes from } \mathrm{n}=6 \\
\text { ind.) }{ }^{a}\end{array}$ & $\checkmark 3, c$ & $++3, c$ & 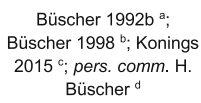 \\
\hline $\begin{array}{l}\text { Neolamprologus brevis }{ }^{4} \\
\text { BOULENGER } 1899\end{array}$ & 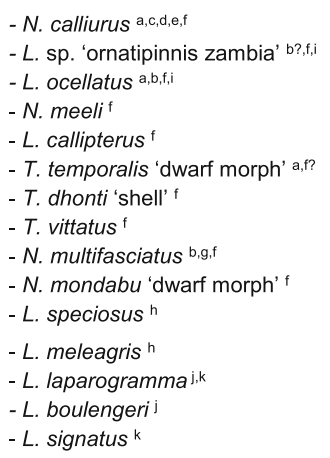 & $\begin{array}{l}3.9-4.4 \mathrm{~cm} \mathrm{SL} \\
\text { (range of averages from } \\
\text { Kombe and Gitaza } \\
\text { populations) }\end{array}$ & $\checkmark a, b, c, f, h$ & $+++\mathrm{a}-\mathrm{e}$ & 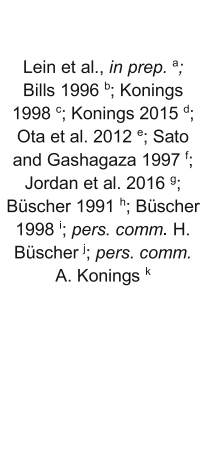 \\
\hline $\begin{array}{l}\text { Neolamprologus calliurus }{ }^{4} \\
\text { BOULENGER } 1906\end{array}$ & $\begin{array}{l}\text { - N. brevis a,c,d,e } \\
\text { - N. multifasciatus a } \\
\text { - T. temporalis 'dwarf morph' a } \\
\text { - L. callipterus }{ }^{a, c, d, d} \\
\text { - T. vittatus }{ }^{a, c, d, e}\end{array}$ & $\begin{array}{l}3.52-6.04 \mathrm{~cm} \mathrm{SL} \\
\text { (Wonzye pop.) }^{5, d}\end{array}$ & $\checkmark c, e$ & $+6, a, d$ & 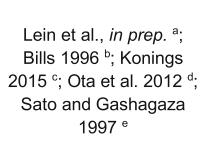 \\
\hline $\begin{array}{l}\text { Neolamprologus mondabu 'shell } \\
\text { morph' } 7 \\
\text { BOULENGER } 1906\end{array}$ & $\begin{array}{l}\text { - L. ocellatus b } \\
\text { - L. callipterus 'shell' a } \\
\text { - N. brevis b } \\
\text { - T. dhonti 'shell' b } \\
\text { - A. sp. 'compressiceps shell' a } \\
\text { (- T. temporalis 'dwarf morph' b?) }\end{array}$ & $\begin{array}{l}3.28-4.82 \mathrm{~cm} \mathrm{SL} \\
\text { (range of sizes from } \mathrm{n}=8 \\
\text { ind.) }{ }^{a}\end{array}$ & $\checkmark 8, \mathrm{a}, \mathrm{b}$ & $++8, a$ & $\begin{array}{c}\text { Gashagaza et al. } 1995^{a} ; \\
\text { Sato and Gashagaza } \\
1997^{\mathrm{b}}\end{array}$ \\
\hline $\begin{array}{l}\text { Lamprologus sp. 'ornatipinnis } \\
\text { zambia' } 9 \\
\text { POLL } 1949\end{array}$ & 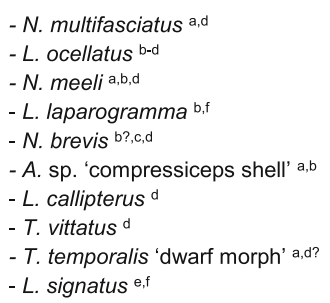 & $\begin{array}{l}3.8-4.3 \mathrm{~cm} \mathrm{SL} \\
\text { (range of averages from } \\
\text { Kombe, Mbita Island, } \\
\text { Musende Rocks and } \\
\text { Gitaza populations) }^{\mathrm{b}}\end{array}$ & $\checkmark$ a-d & $++a, b$ & $\begin{array}{l}\text { Lein et al., in prep. a; } \\
\text { Bills } 1996^{\text {b }} \text {; Büscher } \\
1998^{\text {c; }} \text { Sato and } \\
\text { Gashagaza } 1997^{\text {d; pers. }} \text {. } \\
\text { comm. H. Büscher e; } \\
\text { pers. comm. A. Konings } \\
+\end{array}$ \\
\hline
\end{tabular}


Table 1 continued

\begin{tabular}{|c|c|c|c|c|c|}
\hline \multirow{2}{*}{ Species } & \multirow{2}{*}{ Confirmed syntopy [species] } & \multirow{2}{*}{ Length [cm SL | TL] } & \multicolumn{2}{|c|}{ Shell use } & \multirow{2}{*}{ References } \\
\hline & & & brooding & shelter & \\
\hline $\begin{array}{l}\text { Lamprologus ocellatus } \\
\text { STEINDACHER } 1909\end{array}$ & $\begin{array}{l}\text { - N. brevis a,b,d,f } \\
\text { - N. mondabu 'dwarf morph' d } \\
\text { - N. meeli }{ }^{\mathrm{a}, \mathrm{b}, \mathrm{d}, \mathrm{e}} \\
\text { - L. sp. 'ornatipinnis zambia' a,b,d,f } \\
\text { - T. dhonti }{ }^{\mathrm{d}} \\
\text { - L. callipterus } \mathrm{a}, \mathrm{b}, \mathrm{d} \\
\text { - N. multifasciatus } \mathrm{c}, \mathrm{d} \\
\text { - L. laparogramma }{ }^{\mathrm{b}} \\
\text { - T. vittatus } \mathrm{d} \\
\text { - L. signatus }{ }^{\mathrm{h}} \\
\text { - L. boulengeri } \mathrm{h} \\
\text { - T. temporalis 'dwarf morph' d?,g }\end{array}$ & $\begin{array}{l}3.6-3.9 \mathrm{~cm} \mathrm{SL} \\
\text { (range of averages from } \\
\text { Mbita Isl. and Musende } \\
\text { Rocks populations) }{ }^{b}\end{array}$ & $\checkmark$ a,b,d,e,g & $++10, a, b, e, g$ & 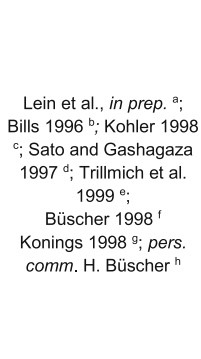 \\
\hline $\begin{array}{l}\text { Lamprologus speciosus } \\
\text { BÜSCHER } 1991\end{array}$ & $\begin{array}{l}\text { - L. meleagris } a, b \\
-N . \text { brevis }^{\mathrm{a}, \mathrm{b}}\end{array}$ & $2.78-3.61 \mathrm{~cm} \mathrm{SL}^{a}$ & $\checkmark 11, \mathrm{a}-\mathrm{c}$ & $++12, a, b$ & $\begin{array}{l}\text { Büscher } 1991 \text { a; Büscher } \\
1998^{\text {b }} \text {; Konings } 2015^{\circ}\end{array}$ \\
\hline $\begin{array}{l}\text { Lamprologus meleagris }{ }^{13} \\
\text { BÜSCHER } 1991\end{array}$ & $\begin{array}{l}\text { - L. speciosus } a, b \\
\text { - N. brevis }{ }^{a, b}\end{array}$ & $\begin{array}{l}2.83-4.28 \mathrm{~cm} \mathrm{SL} \\
\text { (range of sizes from } \mathrm{n}=7 \\
\text { ind.) }{ }^{a}\end{array}$ & $\checkmark a-c$ & $++12, a, b$ & $\begin{array}{l}\text { Büscher } 1991^{\text {a; }} \text {; Büscher } \\
1998^{\text {b }} \text {; Konings } 2015^{c}\end{array}$ \\
\hline $\begin{array}{l}\text { Lamprologus callipterus } \\
\text { BOULENGER } 1906\end{array}$ & 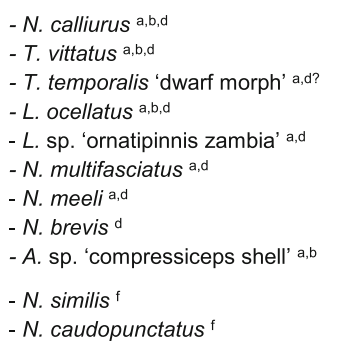 & $3.15-11.6 \mathrm{~cm} \mathrm{SL}{ }^{c, e}$ & $\checkmark$ b-d & $+14, b, c$ & 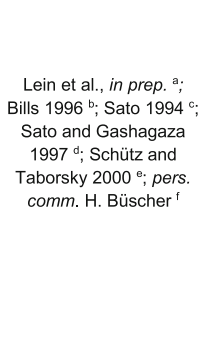 \\
\hline $\begin{array}{l}\text { Neolamprologus meeli }{ }^{15} \\
\text { POLL } 1948\end{array}$ & $\begin{array}{l}\text { - L. ocellatus }{ }^{\mathrm{a}-\mathrm{c}} \\
\text { - T. temporalis 'dwarf morph' a,c?, e } \\
\text { - N. brevis }{ }^{\mathrm{b}, \mathrm{c}} \\
\text { - L. sp. 'ornatipinnis zambia' a-c } \\
\text { - N. multifasciatus } \mathrm{a}, \mathrm{c} \\
\text { - T. vittatus }{ }^{\mathrm{c}} \\
\text { - L. callipterus }{ }^{\mathrm{c}} \\
\text { - A. sp. 'compressiceps shell' a,b } \\
\text { - N. pulcher 'shell' e } \\
\text { - L. boulengeri e }\end{array}$ & $\begin{array}{l}4.31-4.95 \mathrm{~cm} \mathrm{SL} \\
\text { (range of averages from } \\
\text { Kombe, Mbita Isl. and } \\
\text { Musende Rocks } \\
\text { populations) }{ }^{\mathrm{b}} \\
\\
5.74-7.24 \mathrm{~cm} \mathrm{TL} \\
\text { (Wonzye pop.) }^{\mathrm{d}}\end{array}$ & $\checkmark$ b-d & $++a, b, d$ & 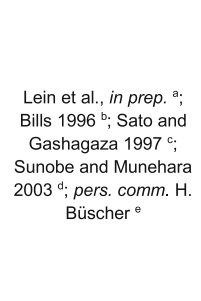 \\
\hline $\begin{array}{l}\text { Telmatochromis temporalis 'dwarf } \\
\text { morph' } 16 \\
\text { BOULENGER } 1898\end{array}$ & $\begin{array}{l}\text { - N. multifasciatus a,b? } \\
\text { - N. meeli } \mathrm{a}, \mathrm{b} \text { ? } \\
\text { - A. sp. 'compressiceps shell' a,b? } \\
\text { - L. ocellatus }{ }^{\mathrm{b} ?, \mathrm{~g}} \\
\text { - L. sp. 'ornatipinnis zambia' a,b? } \\
\text { - N. brevis a,b? } \\
\text { - N. mondabu 'dwarf morph' b? } \\
\text { - L. callipterus a,b? } \\
\text { - T. vittatus } \mathrm{b} \text { ?, } \\
\text { - T. dhonti } \mathrm{b}^{\mathrm{a}} \text { ? } \\
\text { - N. pulcher 'shell' g }\end{array}$ & 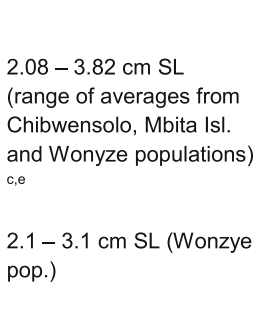 & $\checkmark$ a,b,d,e & $++a, d, e, f$ & $\begin{array}{c}\text { Lein et al., in prep. a; } \\
\text { Sato and Gashagaza } \\
1997^{\text {b; }} \text { Takahashi } 2004 \\
\text { c; Takahashi } 2019^{\text {d; }} \\
\text { Takahashi et al. } 2012 \text { e; } \\
\text { Takahashi et al. } 2009^{f} \\
\text { pers. comm. H. Büscher } \\
\text { g }\end{array}$ \\
\hline $\begin{array}{l}\text { Telmatochromis vittatus } \\
\text { BOULENGER } 1898\end{array}$ & $\begin{array}{l}\text { - L. callipterus }{ }^{\mathrm{a}-\mathrm{e}} \\
-N . \text { meeli }^{\mathrm{e}}\end{array}$ & $\begin{array}{l}<4.5 \mathrm{~cm} \mathrm{TL} \text { female }{ }^{c} \\
2.62 \mathrm{~cm} \mathrm{SL} \mathrm{('sneaker')}\end{array}$ & $\checkmark 17, \mathrm{c}-\mathrm{e}$ & $++18, c$ & $\begin{array}{l}\text { Lein et al., in prep. a; } \\
\text { Bills } 1996 \text { b; Ota and } \\
\text { Kohda } 2006 a^{c} \text {; }\end{array}$ \\
\hline
\end{tabular}


Table 1 continued

\begin{tabular}{|c|c|c|c|c|c|}
\hline \multirow{2}{*}{ Species } & \multirow{2}{*}{ Confirmed syntopy [species] } & \multirow{2}{*}{ Length [cm SL | TL] } & \multicolumn{2}{|c|}{ Shell use } & \multirow{2}{*}{ References } \\
\hline & & & brooding & shelter & \\
\hline (continued) & $\begin{array}{l}\text { - N. calliurus }{ }^{\mathrm{a}-\mathrm{e}} \\
-\mathrm{N} \text {. multifasciatus } \mathrm{a}, \mathrm{e} \\
\text { - T. temporalis 'dwarf morph' a,e?,f } \\
\text { - A. sp. 'compressiceps shell' } \mathrm{a}, \mathrm{b}\end{array}$ & $\begin{array}{l}4.57 \mathrm{~cm} \mathrm{SL} \text { ('satellite') c } \\
5.5 \mathrm{~cm} \mathrm{SL} \text { ('territorial') }^{c} \\
6.41 \mathrm{~cm} \mathrm{SL} \mathrm{('pirate')} \mathrm{c}\end{array}$ & & & $\begin{array}{c}\text { Ota and Kohda 2006b }{ }^{d} \text {; } \\
\text { Sato and Gashagaza } \\
1997^{\text {en }} \text { pers. comm. H. } \\
\text { Büscher }{ }^{f}\end{array}$ \\
\hline $\begin{array}{l}\text { Altolamprologus sp. } \\
\text { 'compressiceps shell' } 19 \\
\text { BOULENGER } 1898\end{array}$ & $\begin{array}{l}\text { - N. multifasciatus a } \\
- \text { T. temporalis 'dwarf morph' a,e? } \\
- \text { T. dhonti 'shell' e } \\
\text { - L. sp. 'ornatipinnis zambia' a } \\
\text { - L. callipterus a } \\
\text { - L. callipterus 'shell' c,e } \\
- \text { T. vittatus a,b } \\
\text { - N. meeli a,b } \\
\text { - L. ocellatus e } \\
- \text { N. brevis } \\
- \text { N. mondabu 'dwarf morph' c,e }\end{array}$ & $\begin{array}{l}3.69-5.7 \mathrm{~cm} \mathrm{SL} \\
\text { (range of sizes from } n=11 \\
\text { ind.) }{ }^{c}\end{array}$ & $\checkmark c-e$ & $++a, c, d$ & $\begin{array}{l}\text { Lein et al., unpub. data } \\
\text { a; Bills } 1996^{\mathrm{b}} \text {; } \\
\text { Gashagaza et al. } 1995^{\mathrm{c}} \text {; } \\
\text { Konings } 2015^{\mathrm{d}} \text {; Sato } \\
\text { and Gashagaza } 1997^{\circ}\end{array}$ \\
\hline $\begin{array}{l}\text { Lamprologus callipterus 'shell } \\
\text { morph' } 20 \\
\text { BOULENGER } 1906\end{array}$ & $\begin{array}{l}\text { - A. sp. 'compressiceps shell' a,b } \\
\text { - N. brevis }{ }^{b} \\
\text { - L. ocellatus b } \\
\text { - T. dhonti 'shell' b } \\
\text { - N. mondabu 'dwarf morph' a,b } \\
\text { (- T. temporalis 'dwarf morph' b?) }\end{array}$ & $\begin{array}{l}4.3-5.11 \mathrm{~cm} \mathrm{SL} \text { (range } \\
\text { of sizes from } n=10 \text { ind.) a }\end{array}$ & $\checkmark \mathrm{a}, \mathrm{b}$ & $+++a$ & $\begin{array}{c}\text { Gashagaza et al. } 1995^{\mathrm{a}} ; \\
\text { Sato and Gashagaza } \\
1997^{\mathrm{b}}\end{array}$ \\
\hline $\begin{array}{l}\text { Neolamprologus sp. "Mwila" } \\
\text { - undescribed species - }\end{array}$ & $\begin{array}{l}\text { - N. meeli a } \\
\text { - L. callipterus a } \\
\text { - T. dhonti a } \\
\text { - N. brevis? a }\end{array}$ & $5-7 \mathrm{~cm} \mathrm{TL}^{\mathrm{a}}$ & $\checkmark$ a & a & $\begin{array}{l}\text { Karlsson \& Karlsson } \\
2020^{\circ}\end{array}$ \\
\hline $\begin{array}{l}\text { Lamprologus laparogramma } \\
\text { BILLS \& RIBBINK } 1997\end{array}$ & $\begin{array}{l}\text { - L. sp. 'ornatipinnis zambia' a,d } \\
-N . \text { meeli a } \\
-N . \text { brevis }^{c, d}\end{array}$ & 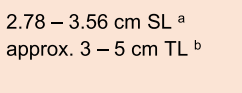 & $\checkmark 21, \mathrm{a}$ & $+21, a$ & 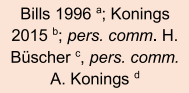 \\
\hline $\begin{array}{l}\text { Lamprologus signatus } \\
\text { POLL } 1952\end{array}$ & $\begin{array}{l}\text { - L. sp. 'ornatipinnis zambia' c,d } \\
\text { - L. ocellatus }{ }^{c} \\
- \text { N. brevis }{ }^{d}\end{array}$ & $\begin{array}{l}2.74-3.57 \mathrm{~cm} \mathrm{SL} \mathrm{a}^{\mathrm{a}} \\
\text { approx. } 3-5 \mathrm{~cm} \mathrm{TL}^{\mathrm{b}}\end{array}$ & $\checkmark 21, \mathrm{a}$ & $+21, a$ & $\begin{array}{l}\text { Bills } 1996^{\text {a }} \text {; Konings } \\
2015^{\text {b}} \text {; pers. comm. H. } \\
\text { Büscher }{ }^{c} \text {, pers. comm. } \\
\text { A. Konings }{ }^{d}\end{array}$ \\
\hline $\begin{array}{l}\text { Neolamprologus pulcher 'shell' } 22 \\
\text { TREWAVAS \& POLL } 1952\end{array}$ & $\begin{array}{l}-T . \text { vittatus }^{\text {a }} \\
-N . \text { meeli }{ }^{\mathrm{b}} \\
\text { - T. temporalis 'dwarf morph' b }\end{array}$ & approx. $<7.5 \mathrm{~cm} \mathrm{TL}$ a & $?^{23}$ & $++23, a$ & $\begin{array}{l}\text { Lein et al., unpub. data } \\
\text { a; pers. comm. H. } \\
\text { Büscher }{ }^{\mathrm{b}}\end{array}$ \\
\hline $\begin{array}{l}\text { Telmatochromis dhonti 'shell } \\
\text { morph' } 24 \\
\text { BOULENGER } 1919\end{array}$ & $\begin{array}{l}\text { - L. callipterus 'shell' a } \\
\text { - N. mondabu 'dwarf morph' a } \\
\text { - A. sp. 'compressiceps shell' a } \\
\text { - N. brevis a } \\
\text { - L. ocellatus a } \\
\text { (- T. temporalis 'dwarf morph' a?) }\end{array}$ & $?$ & $\checkmark$ a & $?$ & Sato \& Gashagaza 1997 \\
\hline $\begin{array}{l}\text { Lepidiolamprologus boulengeri } 25 \\
\text { STEINDACHER } 1909\end{array}$ & $\begin{array}{l}- \text { L. ocellatus }{ }^{b} \\
-N . \text { meeli }^{b} \\
-N . \text { brevis }^{b}\end{array}$ & $5-7 \mathrm{~cm} \mathrm{TL}^{\mathrm{a}}$ & $\checkmark$ a & $+26, a$ & $\begin{array}{l}\text { Konings } 2015^{\text {a }} \text {; pers. } \\
\text { comm. H. Büscher }{ }^{b}\end{array}$ \\
\hline $\begin{array}{l}\text { Neolamprologus caudopunctatus } \\
\text { POLL } 1978\end{array}$ & $\begin{array}{l}\text { - L. callipterus }{ }^{a} \\
-T . \text { vittatus }^{a} \\
-N . \text { multifasciatus }^{d} \\
-N . \text { similis }^{d}\end{array}$ & $\begin{array}{c}4.45-5.05 \mathrm{~cm} \mathrm{SL}^{\text {a }} \\
\text { (Mbita pop.) } \\
\\
6.5-7.5 \mathrm{~cm} \mathrm{TL}^{\mathrm{b}} \\
4.5-6 \mathrm{~cm} \mathrm{TL}^{\mathrm{c}} \\
\text { (Kasakalawe pop.) }\end{array}$ & $\checkmark b$ & $?$ & $\begin{array}{c}\text { Ochi and Yanagisawa } \\
1999^{\text {a; }} \\
\text { Schädelin et al. } 2012^{\text {b; }} \\
\text { Schädelin et al. } 2015^{\text {c; }} \\
\text { pers. comm. H. Büscher } \\
\text { d }\end{array}$ \\
\hline $\begin{array}{l}\text { Neolamprologus wauthioni }{ }^{27} \\
\text { POLL } 1949\end{array}$ & $?$ & $?$ & $?$ & $?$ & Konings 2015 \\
\hline
\end{tabular}


Table 1 continued

\begin{tabular}{l}
\hline (With)in some populations of $N$. multifasciatus (e.g. Mbete Bay, Cape Kapembwa, ZA) individuals are also known to brood and \\
hide in cracks or in-between stones (Kohler 1998, pers. comm. H. Büscher) \\
${ }^{2}$ Not explicitly stated; based on combined information on geographical and depth distribution (Büscher 1992b, Büscher 1998) \\
${ }^{3}$ Some populations of $N$. similis (Mbete Bay, Cape Kapembwa; Kohler 1998) are known to brood and hide in cracks or in-between \\
stones (Büscher 1992b) \\
${ }^{4} N$. brevis and $N$. calliurus are usually treated as two separate species (Sturmbauer et al., 1994, Koblmüller et al., 2007, Konings \\
2015 , Ronco et al., 2019), but Ota et al., (2012) see in what has been described as $N$. brevis the sub-adult stage of $N$. calliurus (and \\
refer to both as $N$. brevis). These two species are referred to as $N$. brevis ("out of the nest [of L. callipterus]"), respectively as $N$.
\end{tabular} brevis ("in the nest [of L. callipterus]"), in Sato and Gashagaza (1997)

${ }^{5}$ In their analysis of body sizes Ota et al., (2012) refer to these as individuals from shell beds/separated shells on sand bottom and individuals in shell patches/in midwater aggregations, which we interpret to refer to members of the species $N$. brevis and $N$. calliurus, respectively

${ }^{6}$ Due to the large size of males shells are used as shelter mainly by females (Ota et al., 2012)

7 The attribute 'shell morph' was chosen here to distinguish this population, that had been originally described by Gashagaza et al., (1995), from the sand/rock-dwelling $N$. mondabu populations

${ }^{8} \mathrm{~N}$. mondabu females dig a hole at the side of Neothauma shells and spawn their eggs on the shells' outer surface; the hole also serves as shelter. Territorial males use shells as shelters (Gashagaza et al., 1995)

${ }^{9}$ According to Konings (2015)

${ }^{10}$ When no shells are immediately accessible, to escape predators this species might instead dive into the sandy bottom (Konings 2015)

${ }^{11}$ Not stated explicitly

12 When no shells are immediately accessible, to escape predators this species might instead dive into the sandy bottom (Konings 2015, pers. comm. H. Büscher) or flee (Büscher 1991)

${ }^{13}$ It is suspected that L. meleagris and L. stappersi are indeed the same species (Konings 2015). Since for L. stappersi PELLEGRIN 1927 only the holotype exists in museum collections, comparisons to other taxa are difficult (Ronco et al., 2019)

${ }^{14}$ Due to the large size of territorial males, shells are exclusively used by females and certain types of males with alternative reproductive strategies (Sato 1994)

15 Also called Lepidiolamprologus meeli / Lamprologus meeli

${ }^{16}$ It has been demonstrated that $T$. temporalis 'dwarf morph' is a genetically distinct dwarf-sized ecomorph of the rock-dwelling $T$. temporalis (Takahashi 2004; Takahashi et al., 2009; Winkelmann et al., 2014). At the time Sato \& Gashagaza published their work on shell-brooding cichlids (1997), the status of this species was still under investigation. It is therefore unclear whether the authors had observed subadult rock-dwelling individuals, or alternatively, adult dwarf-size individuals at the study locations (denoted with a superscript "?" in the "Confirmed syntopy [species]" column)

${ }^{17}$ Spawning might also occur in small crevices (Ota \& Khoda 2006a)

18 Only small individuals hide in shells (Ota \& Khoda 2006a)

${ }^{19}$ Listed as currently potentially undescribed species in Ronco et al., (2019)

${ }^{20}$ The attribute 'shell morph' was chosen here to distinguish this population, that had been originally described by Gashagaza et al., (1995) and in which also territorial males are small enough to enter empty Neothauma shells, from the standard L. callipterus

${ }^{21}$ L. laparogramma and L. signatus typically live in holes which are dug into the muddy substrate, but when shells are abundant in muddy habitats they also make use of them as shelters and breeding sites (Bills 1996)

${ }^{22}$ Known to occur at Mwina, Mutondwe Island, Zambia (Lein et al., unpub. data; pers. comm. H. Büscher). Usually N. pulcher broods and seeks shelter between and underneath flat and small round stones that are maintained through digging (Balshine et al., 2001; Taborsky et al., 2005)

${ }^{23}$ The N. pulcher Mwina population constructs their nests from stone bricks as well as from empty Neothauma shells (Lein et al.," unpub. data). Observations in the Lake, however, suggest that the fish only occasionally access these shells and instead mainly use the interstitial space (pers. comm. H. Büscher)

24 The attribute 'shell morph' was chosen here to distinguish this population from the rock-dwelling T. dhonti populations

25 Also called Neolamprologus boulengeri

${ }^{26}$ Females of this species use shells only sometimes as shelters, whereas males flee over the sand when threatened (Konings 2015)

${ }^{27}$ Originally described as Lamprologus wauthioni 
(Kornfield \& Smith, 2000), has received arguably the most attention by ethologists. Notably, the overwhelming majority of all recognized and suspected cases of cooperative breeding in fish (76-84\%) can be found within this tribe (Taborsky, 1994; Heg \& Bachar, 2006), which comprises 87 valid lacustrine species to date (Ronco et al., 2019), making Lamprologine cichlids a model system for the integrative study of social behaviour (Jordan et al., 2020).

\section{Shell use as a unifying trait}

Unique to the substrate-breeding tribe of Lamprologini, a number of species have specialized in the use of empty snail shells (Koblmüller et al., 2007; Sato \& Gashagaza, 1997; Fig. 1). Due to Lake Tanganyika's alkaline waters (De Wever et al., 2005) that prevent their rapid dissolution, the calcareous shells of molluscs of the genus Neothauma accumulate in extensive layers on the lake benthos (Cohen \& Thouin, 1987), where they are used by a number of cichlid species as brooding chambers and/or as shelters from predators or conspecifics (Table 1). While Lamprologine cichlids are morphologically the most diverse tribe of Tanganyikan cichlids (Clabaut et al., 2007), most cichlids permanently dwelling in shells, confined by the snail shell's morphology, exhibit close resemblance in body size (Table 1), which is regarded as a direct adaptation to this specific life style (Gashagaza et al., 1995; Sato \& Gashagaza, 1997; Büscher, 1998; Kohler, 1998; Takahashi \& Ota, 2016).

\section{Diet}

Earlier studies that have analysed stomach contents in a selection of shell-dwelling cichlid species suggest that many species in question predominantly prey on small benthic invertebrates and zooplankton (Table 2). The conclusion that these food items constitute an important dietary staple for these small-sized cichlids is corroborated by earlier work which suggests that Lamprologines are carnivorous (Hori, 1983; Gashagaza \& Nagoshi, 1986). Further support derives from behavioural observations in the natural habitat, which indicate that a majority of shelldwelling cichlids (including the species previously examined for stomach contents) exhibit functionally and kinematically similar foraging behaviour, involving either picking small prey from the substrate and/or

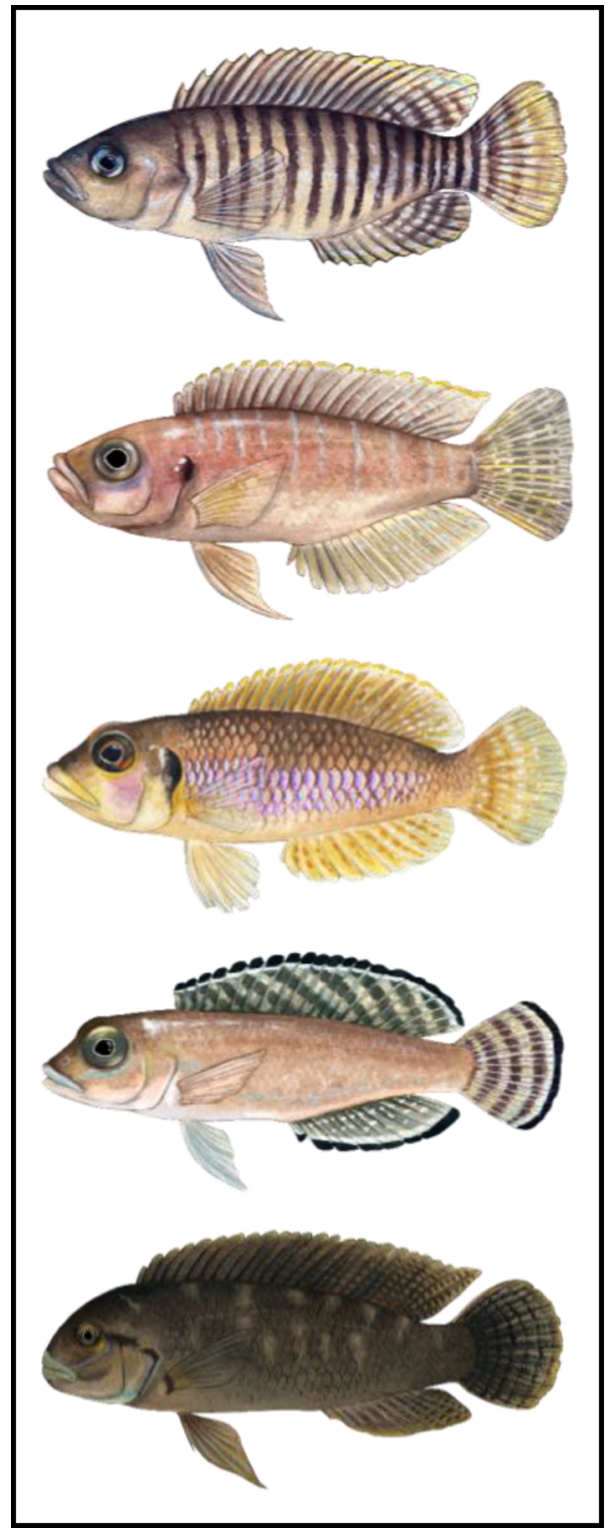

Fig. 1 Shell-dwelling Lamprologine cichlids. Neolamprologus multifasciatus, Neolamprologus brevis, Lamprologus ocellatus, Lamprologus ornatipinnis and Telmatochromis temporalis 'dwarf morph' (top to bottom; not to scale). Illustrations by Alexandra Viertler, courtesy of Jordan Lab.

snatching them directly from the water column (Table 2). The case of Lamprologus sp. 'ornatipinnis zambia', a species with both sex-specific and large seasonal shifts in the consumed diet (Fryer \& Iles, 1972; Gordon \& Bills, 1999), demonstrates the caution that must be taken when interpreting results on small sample sizes and those of unknown specific collection 
Table 2 Mating systems and diets of permanently shell-dwelling cichlids; light red shaded cells denote further potential candidate species for shell-dwelling, albeit with currently sparse information

\begin{tabular}{|c|c|c|c|c|c|}
\hline \multirow{2}{*}{ Species } & \multirow{2}{*}{ Mating system } & \multicolumn{3}{|c|}{ Diet } & \multirow{2}{*}{ References } \\
\hline & & gut content & jaw morphology & feeding behaviour & \\
\hline $\begin{array}{l}\text { Neolamprologus } \\
\text { multifasciatus } \\
\text { BOULENGER } 1906\end{array}$ & $\begin{array}{l}\text { polygyny/polyandry/ } \\
\text { polygynandry a,b,e }\end{array}$ & $\begin{array}{l}\text { mainly copepods, } \\
\text { microfilamentous and } \\
\text { unicellular algae, other small } \\
\text { invertebrates }^{\text {b }}\end{array}$ & $\begin{array}{l}\text { relatively large } \\
\text { canines in upper } \\
\text { and lower jaw } 1, b\end{array}$ & $\begin{array}{l}\text { snatching by- } \\
\text { drifting (zoo-) } \\
\text { plankton b,c,d,f } \\
\text { (copepods); picking } \\
\text { benthic } \\
\text { invertebrates }{ }^{c, f}\end{array}$ & $\begin{array}{c}\text { Bose, submitted. a; } \\
\text { Kohler } 1998^{\text {b; }} \text { Konings } \\
2015^{\text {c; }} \text { Rossiter } 1993^{\text {d; }} \\
\text { Schradin and Lamprecht } \\
2002^{\text {e; }} \text { Lein et al., in } \\
\text { prep. }{ }^{f}\end{array}$ \\
\hline $\begin{array}{l}\text { Neolamprologus similis } \\
\text { BÜSCHER } 1992\end{array}$ & $\begin{array}{l}\text { polygyny, } \\
\text { polyandry or } \\
\text { polygynandry a }\end{array}$ & $\begin{array}{c}\text { copepods, crustaceans, } \\
\text { insect larvae, diatoms, algae } \\
\text { b }\end{array}$ & $?$ & $\begin{array}{l}\text { snatching } \\
\text { zooplankton } \\
\text { (copepods) }{ }^{c} \text {; } \\
\text { picking benthic } \\
\text { invertebrates }^{c}\end{array}$ & $\begin{array}{l}\text { pers. comm. H. Büscher } \\
\text { to Heg and Bachar } 2006 \\
\text { a; Büscher } 1992 b^{b} \text {; } \\
\text { Konings } 2015^{c} \text {; }\end{array}$ \\
\hline $\begin{array}{l}\text { Neolamprologus brevis }{ }^{2} \\
\text { BOULENGER } 1899\end{array}$ & $\begin{array}{l}\text { monogamy - } \\
\text { bigamy } 3, a, e, f\end{array}$ & copepods $\mathrm{b,d,g}$ & $?$ & $\begin{array}{l}\text { snatching by- } \\
\text { drifting plankton a-c; } \\
\text { feeding on } \\
\text { invertebrates }^{\text {a }}\end{array}$ & 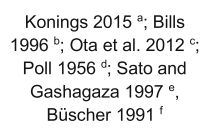 \\
\hline $\begin{array}{l}\text { Neolamprologus calliurus }{ }^{2} \\
\text { BOULENGER } 1906\end{array}$ & $\begin{array}{c}\text { polygyny/ } \\
\text { polygynandry }{ }^{3, a-c}\end{array}$ & $?$ & $?$ & $\begin{array}{c}\text { snatching of by- } \\
\text { drifting zooplankton } \\
\text { a,b; } \\
\text { feeding on } \\
\text { invertebrates }^{\text {a }}\end{array}$ & $\begin{array}{l}\text { Konings } 2015^{\mathrm{a}} ; \\
\text { Ota et al. } 2012^{\mathrm{b}} ; \\
\text { Sato and } \\
\text { Gashagaza } 1997^{\circ}\end{array}$ \\
\hline $\begin{array}{l}\text { Neolamprologus mondabu } \\
\text { 'shell morph' } 4 \\
\text { BOULENGER } 1906\end{array}$ & polygyny a & $\begin{array}{l}\text { Chironomidae? copepods? } \\
\text { b }\end{array}$ & $?$ & $\begin{array}{l}\text { picking prey from } \\
\text { the substrate } \\
\text { surface or sediment } \\
5, b\end{array}$ & $\begin{array}{c}\text { Gashagaza et al. } 1995^{\text {a }} ; \\
\text { Yuma } 1994^{\mathrm{b}}\end{array}$ \\
\hline $\begin{array}{l}\text { Lamprologus sp. 'ornatipinnis } \\
\text { zambia' } 6 \\
\text { POLL } 1949\end{array}$ & $\begin{array}{c}\text { monogamy a,b,e - } \\
\text { bigamy }^{e} \text { - polygyny } \\
\text { a,b,d }\end{array}$ & $\begin{array}{l}\text { Chironomidae, copepods, } \\
\text { ostracods }^{c}\end{array}$ & $\begin{array}{l}\text { jaw: enlarged } \\
\text { canines }{ }^{c} \\
\text { pharyngeal jaw: } \\
\text { suggests benthic } \\
\text { arthropod feeder }{ }^{c}\end{array}$ & $\begin{array}{c}\text { picking invertebrate } \\
\text { prey from substrate } \\
\text { surface or sediment } \\
\qquad a, b\end{array}$ & 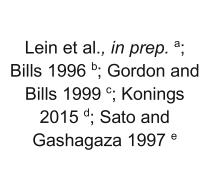 \\
\hline $\begin{array}{l}\text { Lamprologus ocellatus } \\
\text { STEINDACHER } 1909\end{array}$ & $\begin{array}{l}\text { monogamy a,b,c,e } \\
\text { polygyny }{ }^{a-f}\end{array}$ & Chironomidae, ostracods ${ }^{c}$ & $?$ & $\begin{array}{l}\text { picking invertebrate } \\
\text { prey from substrate } \\
\text { surface or sediment } \\
\text { a,b,d; snatching of } \\
\text { by-drifting } \\
\text { zooplankton d }\end{array}$ & 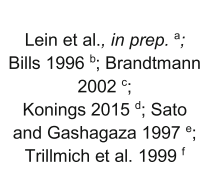 \\
\hline $\begin{array}{l}\text { Lamprologus speciosus } \\
\text { BÜSCHER } 1991\end{array}$ & polygyny a & $\begin{array}{c}\text { mainly insect larvae } \\
\text { (Chironimidae); copepods }{ }^{b}\end{array}$ & $?$ & $\begin{array}{l}\text { picking invertebrate } \\
\text { prey (insect larvae, } \\
\text { small shrimps) from } \\
\text { substrate surface; } \\
\text { snatching by- } \\
\text { drifting plankton a }\end{array}$ & $\begin{array}{c}\text { Konings } 2015^{\mathrm{a}} \text {; Büscher } \\
1991^{\mathrm{b}}\end{array}$ \\
\hline
\end{tabular}


Table 2 continued

\begin{tabular}{|c|c|c|c|c|c|}
\hline \multirow{2}{*}{ Species } & \multirow{2}{*}{ Mating system } & \multicolumn{3}{|c|}{ Diet } & \multirow{2}{*}{ References } \\
\hline & & gut content & jaw morphology & feeding behaviour & \\
\hline $\begin{array}{l}\text { Lamprologus meleagris }{ }^{7} \\
\text { BÜSCHER } 1991\end{array}$ & polygyny a & $\begin{array}{c}\text { mainly insect larvae } \\
\text { (Chironimidae); copepods }\end{array}$ & $?$ & $\begin{array}{l}\text { picking invertebrate } \\
\text { prey (insect larvae, } \\
\text { small shrimps) from } \\
\text { substrate surface; } \\
\text { snatching by- } \\
\text { drifting plankton a }\end{array}$ & $\begin{array}{c}\text { Konings } 2015^{\mathrm{a}} \text {; Büscher } \\
1991^{\mathrm{b}}\end{array}$ \\
\hline $\begin{array}{l}\text { Lamprologus callipterus } \\
\text { BOULENGER } 1906\end{array}$ & polygyny a,b & shrimp, copepods b,c & $?$ & $\begin{array}{l}\text { picking shrimp from } \\
\text { the substrate } \\
\text { surface or sediment } \\
\text { c; feeding on } \\
\text { shrimp and fry }{ }^{c}\end{array}$ & 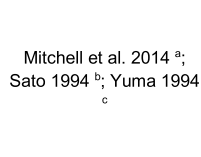 \\
\hline $\begin{array}{l}\text { Neolamprologus meeli }{ }^{8} \\
\text { POLL } 1948\end{array}$ & $\begin{array}{l}\text { monogamy- }{ }^{a-c} \\
\text { polygyny }{ }^{9,10, b, c}\end{array}$ & $?$ & $?$ & $\begin{array}{l}\text { picking invertebrate } \\
\text { prey from substrate } \\
\text { surface or sediment } \\
\text { a; feeds from } \\
\text { hovering position } \\
\text { over the substrate } \\
\text { a,b; occasionally } \\
\text { preying on small } \\
\text { shell-dwellers }{ }^{a}\end{array}$ & $\begin{array}{l}\text { Bills } 1996 \text { a; Konings }{ }^{b} \text {; } \\
\text { Sunobe and Munehara } \\
\qquad 2003^{c}\end{array}$ \\
\hline $\begin{array}{l}\text { Telmatochromis temporalis } \\
\text { 'dwarf morph' } 11 \\
\text { BOULENGER } 1898\end{array}$ & polygyny a & $?$ & $\begin{array}{l}\text { six anteriormost } \\
\text { teeth on } \\
\text { premaxillae } \\
\text { enlarged; small } \\
\text { lateral teeth }{ }^{b}\end{array}$ & $\begin{array}{l}\text { feeding of epilithic } \\
\text { algae }^{a}\end{array}$ & $\begin{array}{l}\text { Takahashi et al. } 2012^{\text {a }} \text {; } \\
\text { Takahashi } 2004^{\mathrm{b}}\end{array}$ \\
\hline $\begin{array}{l}\text { Telmatochromis vittatus } \\
\text { BOULENGER } 1898\end{array}$ & polygyny? a & $?$ & $?$ & $\begin{array}{c}\text { feeding on } \\
\text { filamentous algae } \\
\text { and/or zooplankton } \\
\text { b; eggs of } \\
\text { heterospecifics }^{c}\end{array}$ & $\begin{array}{c}\text { Ota and Kohda 2006b a; } \\
\text { pers. comm. M. Hori to } \\
\text { Ota and Kohda 2006a } \\
\text { Bruintjes \& Taborsky } \\
2011^{c}\end{array}$ \\
\hline $\begin{array}{l}\text { Altolamprologus sp. } \\
\text { 'compressiceps shell' } 12 \\
\text { BOULENGER } 1898\end{array}$ & polygyny? ${ }^{12, \text { a }}$ & shrimp? ${ }^{13, b}$ & $?$ & $\begin{array}{l}\text { picking shrimp from } \\
\text { the substrate } \\
\text { surface }{ }^{13, b}\end{array}$ & $\begin{array}{c}\text { Konings } 2015^{\text {a }} \text {; Yuma } \\
1994^{\mathrm{b}}\end{array}$ \\
\hline $\begin{array}{l}\text { Lamprologus callipterus } \\
\text { 'shell morph' } 14 \\
\text { BOULENGER } 1906\end{array}$ & polygyny a & shrimp? copepods? ${ }^{15, b, c}$ & $?$ & $\begin{array}{c}\text { picking shrimp from } \\
\text { the substrate } \\
\text { surface or sediment } \\
15, c\end{array}$ & 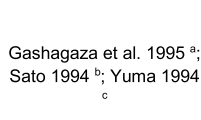 \\
\hline $\begin{array}{l}\text { Neolamprologus sp. "Mwila" } \\
\text { - undescribed - }\end{array}$ & $?$ & $?$ & $?$ & $?$ & $\begin{array}{c}\text { Karlsson \& Karlsson } \\
2020\end{array}$ \\
\hline $\begin{array}{l}\text { Lamprologus laparogramma } \\
\text { BILLS \& RIBBINK } 1997\end{array}$ & monogamy $a, b$ & $?$ & large canines ${ }^{b}$ & $\begin{array}{c}\text { picking of } \\
\text { zooplankton and } \\
\text { small invertebrates } \\
b, c\end{array}$ & 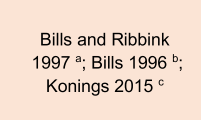 \\
\hline $\begin{array}{l}\text { Lamprologus signatus } \\
\text { POLL } 1952\end{array}$ & monogamy $a, b$ & $?$ & large canines ${ }^{b}$ & $\begin{array}{l}\text { picking of zoo- } \\
\text { plankton and } \\
\text { small in- } \\
\text { vertebrates }\end{array}$ & $\begin{array}{l}\text { Bills and Ribbink } 1997^{\text {a }} \\
\text { Bills } 1996^{\mathrm{b}} ; \\
\text { Konings } 2015^{\mathrm{c}}\end{array}$ \\
\hline
\end{tabular}


Table 2 continued

\begin{tabular}{|c|c|c|c|c|c|}
\hline \multirow{2}{*}{ Species } & \multirow{2}{*}{ Mating system } & \multicolumn{3}{|c|}{ Diet } & \multirow{2}{*}{ References } \\
\hline & & gut content & jaw morphology & feeding behaviour & \\
\hline (continued) & & & & $\begin{array}{l}\text { feeding from a } \\
\text { resting position on } \\
\text { the substrate }\end{array}$ & \\
\hline $\begin{array}{l}\text { Neolamprologus pulcher } \\
\text { 'shell' } 16 \\
\text { TREWAVAS \& POLL } 1952\end{array}$ & $\begin{array}{l}\text { monogamy }{ }^{a, d, e}- \\
\text { polygyny } 17, a, d, e\end{array}$ & copepods? ${ }^{17, \mathrm{c}}$ & $?$ & $\begin{array}{c}\text { snatching of by- } \\
\text { drifting zooplankton } \\
f\end{array}$ & $\begin{array}{c}\text { Desjardins et al. } 2008^{\text {a; }} \\
\text { Gashagaza } 1988^{\text {b; }} \\
\text { Gashagaza and Nagoshi } \\
\text { 1985b c; Limberger } 1983 \\
\text { d; Taborsky and } \\
\text { Limberger } 1981^{\mathrm{e}} ; \text { pers. } \\
\text { comm. H. Büscher }\end{array}$ \\
\hline $\begin{array}{l}\text { Telmatochromis dhonti 'shell } \\
\text { morph' }{ }^{18} \\
\text { BOULENGER } 1919\end{array}$ & $\begin{array}{c}\text { monogamy }{ }^{a}- \\
\text { polygyny a }\end{array}$ & $\begin{array}{l}\text { invertebrates? midge larvae? } \\
\qquad 19, b\end{array}$ & $?$ & $?$ & $\begin{array}{l}\text { Sato \& Gashagaza } 1997 \\
\quad \text { a; Konings } 2015^{b}\end{array}$ \\
\hline $\begin{array}{l}\text { Lepidiolamprologus } \\
\text { boulengeri }{ }^{20} \\
\text { STEINDACHER } 1909\end{array}$ & $\begin{array}{l}\text { monogamy- } \\
\text { polygyny }^{a}\end{array}$ & $?$ & $?$ & $?$ & Konings $2015^{\text {a }}$ \\
\hline $\begin{array}{l}\text { Neolamprologus } \\
\text { caudopunctatus } \\
\text { POLL } 1978\end{array}$ & monogamy a,b & $\begin{array}{l}\text { ostracods, insect larvae, } \\
\text { copepods, algae }{ }^{d}\end{array}$ & $?$ & $\begin{array}{c}\text { picking } \\
\text { invertebrates from } \\
\text { the substrate } \\
\text { surface and water } \\
\text { column }{ }^{c}\end{array}$ & $\begin{array}{c}\text { Schädelin et al. } 2012 \text { a; } \\
\text { Schädelin et al. } 2015^{\mathrm{b}} \text {; } \\
\text { Konings } 2015^{\text {c } ; \text { Büscher }} \\
\text { 1992a }{ }^{\mathrm{d}}\end{array}$ \\
\hline $\begin{array}{l}\text { Neolamprologus wauthioni }{ }^{21} \\
\text { POLL } 1949\end{array}$ & $?$ & $?$ & $?$ & $?$ & Konings 2015 \\
\hline
\end{tabular}

${ }^{1}$ Kohler (1998) suspects that these large canines are possibly connected with intra- and inter-specific conflict

${ }^{2}$ N. brevis and N. calliurus are usually treated as two separate species (Sturmbauer et al., 1994, Koblmüller et al., 2007, Konings 2015, Ronco et al., 2019), but Ota et al., (2012) see in what has been described as $N$. brevis the sub-adult stage of N. calliurus (and refer to both as $N$. brevis). These two species are referred to as N. brevis ("out of the nest [of L. callipterus]"), respectively, as $N$. brevis ("in the nest [of L. callipterus]"), in Sato \& Gashagaza (1997)

${ }^{3}$ Sato \& Gashagaza (1997) describe the mating system as mono-bigamous for those individuals that are found outside the nest of $L$. callipterus, and polygamous for those individuals that are found inside the nest, which we interpret to refer to members of the species $N$. brevis and N. calliurus, respectively

${ }^{4}$ The attribute 'shell morph' was chosen here to distinguish this population that had been originally described by Gashagaza et al., (1995), from the sand/rock-dwelling N. mondabu populations

${ }^{5}$ Information based on different (non-shell-dwelling) populations

${ }^{6}$ According to Konings (2015)

${ }^{7}$ It is suspected that L. meleagris and L. stappersi are indeed the same species (Konings 2015). Since for L. stappersi PELLEGRIN 1927 only the holotype exists in museum collections, comparisons to other taxa are difficult (Ronco et al., 2019)

${ }^{8}$ Also called Lepidiolamprologus meeli / Lamprologus meeli

${ }^{9}$ Sunobe \& Munehara (2003) refer to this species as Neolamprologus meeli

${ }^{10}$ Bills (1996) refers to this species as Neolamprologus hecqui

${ }^{11}$ It has been demonstrated that $T$. temporalis 'dwarf morph' is a genetically distinct dwarf-sized ecomorph of the rock-dwelling $T$. temporalis (Takahashi 2004; Takahashi et al., 2009; Winkelmann et al., 2014)

12 Listed as currently potentially undescribed species in Ronco et al., (2019)

${ }^{13}$ Information based on different (non-shell-dwelling) populations

14 The attribute 'shell morph' was chosen here to distinguish this population that had been originally described by Gashagaza et al. (1995) and in which also territorial males are small enough to enter empty Neothauma shells, from the standard L. callipterus 
Table 2 continued

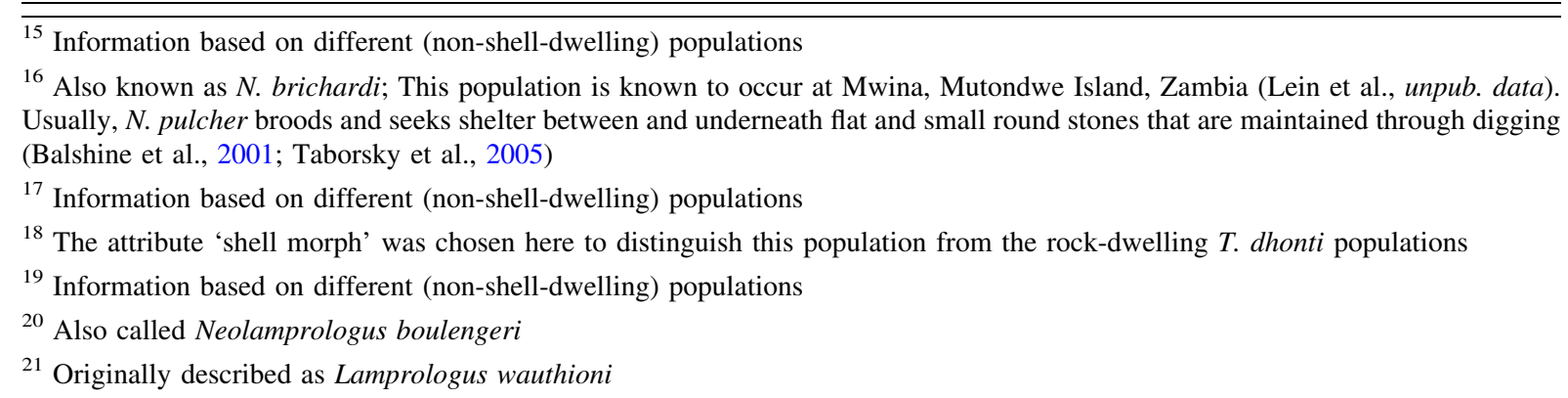

conditions. Like all members of the family Cichlidae, however, Tanganyika cichlids possess pharyngeal jaws which exhibit strong phenotypic plasticity, and pharyngeal jaw morphology is known to strongly correlate with dietary preferences (Takahashi and Koblmüller 2011), making morphometrical analyses a helpful piece of information when drawing conclusions with respect to individual, population, and species-level dietary niche. However, as of now, data on pharyngeal jaw morphology are relatively sparse, and inferences based on stable isotopes are a similarly effective proxy for assessing dietary niches. Stable isotope analyses conducted on fifteen different shelldwelling cichlid species demonstrate broadly similar trophic niches (F. Ronco, W. Salzburger, in preparation), although there are notable differences for some species (e.g. N. similis having considerably lower $\delta^{15} \mathrm{~N}$ than $L$. sp. 'ornatipinnis zambia'). Since brain size and overall head shape are phenotypically integrated in Tanganyikan cichlids, and head shape is correlated with feeding mode (Tsuboi et al., 2014), a system exhibiting only little variation in feeding ecology is of importance when studying brain evolution. Overall, current data confirm the general assertion that shelldwelling cichlids share the same trophic level, relatively similar dietary preferences, and functionally closely related foraging modes.

\section{Distribution}

Most shell-dwelling cichlids are found syntopically (i.e. occupying the same macro-habitat, occurring together in the same locality, and being observable in close spatial proximity; Rivas, 1964) with some others occurring at least sympatrically (see Table 1 for specific details; Koblmüller et al., 2007; Sato \&
Gashagaza, 1997). Shell-dwelling cichlids occupy physical environments ranging from muddy or sandy substrates interspersed with few gastropod shells (e.g. Lamprologus ocellatus), to rubble-covered substrate with small localized clusters of shells (e.g. Neolamprologus brevis), up to so-called "shell beds" which are extensive areas composed entirely of empty shells (e.g. Neolamprologus multifasciatus; Sato \& Gashagaza, 1997). These differences in micro-habitat structure could represent a source of variation in habitat complexity (Gutiérrez et al., 2003) with potential effects in the social domain (Shumway, 2010; Shumway et al., 2007). Indeed, shell beds and localized clusters of shells appear to harbour high densities of individuals as well as a relatively high species diversity per unit of area (Sato \& Gashagaza, 1997), and thus represent a potential confounding effect, yet as we detail below, this can be partially alleviated ( $\S$ "Outstanding challenges in the study of social and cognitive evolution").

\section{Predation}

Species of similar size living syntopically, like the shell-dwelling cichlids, likely face a similar set of heterospecific predators in their environment (Fryer \& Iles, 1972). This suite of predators may fluctuate with regard to abundances and proportions, resulting from density-related effects on the micro-habitat level with consequences for predator encounter rates (Wrona \& Dixon, 1991). Different populations of the Lamprologine cichlid $N$. pulcher, for instance, have been reported to face different predation pressures, which in turn are reflected in changes in social group structure (Groenewoud et al., 2016). While this source of variation implies a potential confounding factor (van 
der Bijl \& Kolm, 2016)-particularly when comparing across non-sympatric species-a logical remedy is to focus investigations specifically to those shell-dwelling species that occur within overlapping, mosaic communities (for example, cf. Sato \& Gashagaza, 1997). Alternatively, or in addition to this, different populations of the same species at different localities allow the quantification of the effect of these sources of variation and an explicit examination of their significance for cognitive divergence among populations.

\section{Diverse and highly developed social and cognitive capacities}

\section{Sophisticated social behaviours}

Cichlid social behaviour-involving courtship displays and pair-bond reinforcement, numerous different submissive, affiliative, and aggressive behaviours exhibited during contests-encompasses an immense range of behaviours, contexts, and communicatory channels (Jordan et al., 2020). It was the wealth of behaviours observed in captive cichlids that caught the attention of early ethologists (Noble, 1937; Noble \& Curtis, 1939; Aronson, 1949; Baerends \& Baerendsvan Roon, 1950; El-Zarka, 1956; Wickler, 1962), who created ethograms rivalling those of mammals in their behavioural sophistication and diversity. Current ethograms of Lamprologines, such as those for the cooperatively breeding cichlid $N$. pulcher and the shell-dwelling cichlid $N$. multifasciatus, contain between 13 and 18, respectively 12, distinct visually observable social behaviours (Kohler, 1998; Sopinka et al., 2009; Taves et al., 2009; Lein et al., in prep.), affording an inspection of the social relationships that individuals within these species share at a fine-grained level.

\section{Cognitive capacity}

Despite their reputation as relatively primitive vertebrates, a number of fish species, including species from the tribes of Lamprologini and closely related Haplochromini, have been demonstrated to possess a range of sophisticated cognitive skills. These involve the ability to (individually) recognize conspecifics ( $N$. pulcher: Hert, 1985; Balshine-Earn \& Lotem, 1998;
Frostman \& Sherman, 2004; Kohda et al., 2015; Saeki et al., 2018; Julidochromis transcriptus: Hotta et al., 2017; Astatotilapia burtoni: (Weitekamp \& Hofmann, 2017), to memorize socially relevant information for multiple days (J. transcriptus: Hotta et al., 2014) and to use experience as a way to modulate social behaviour during contests (A. burtoni: Alcazar et al., 2014; N. pulcher: Fischer et al., 2015). Furthermore, it has been shown that members of these cichlid tribes possess the ability to create mental representations of hierarchies (J. transcriptus: Hotta et al., 2015a; Hotta et al., 2015b; A. burtoni: Grosenick et al., 2007), the ability to tactically deceive conspecifics (A. burtoni: Desjardins et al., 2012) or redirect aggression to a third party as a form of conflict management (Julidochromis regani: Ito et al., 2018). While it still remains to be tested to which extent the shell-dwelling cichlids match their close relatives in this striking level of cognitive capacity, numerous teleost fish have evolved a suite of adaptations to acquire, process, store, and act on information (definition by Shettleworth, 2009), cognitive abilities comparable to those of birds and mammals (Bshary et al., 2002; Brown et al., 2011; for a review on fish social cognition see Bshary et al., 2014). Cichlids, therefore, appear particularly well suited to investigations into the coevolution of sociality and cognition (Bshary et al., 2002; Bshary and Brown 2014).

Neuroanatomy and an evolutionary conserved "social brain"

Brain regions considered relevant for social behaviour are conserved across the five major vertebrate lineages, including mammals and teleost fish (O'Connell \& Hofmann, 2011; O'Connell \& Hofmann, 2012). Of crucial importance in this context are two neural circuits, the Social Behaviour Network (SBN; Newman, 1999; Goodson, 2005) and the mesolimbic reward system. Together these circuits integrate into the higher order social decision-making network (SDMN) where stimulus salience is evaluated, and where adaptive social behaviours including reproduction, aggression, and parental care are regulated (O'Connell \& Hofmann, 2012). Although the proposed homology relationships for most of SDMNassociated nuclei do not necessarily imply conserved function (Goodson and Kingsbury 2013), the SDMN regions are logical targets for investigating 
mechanisms of cognitive evolution in response to social pressures in cichlids. To that end, as a first step, representative histological brain atlases are currently being constructed on the basis of referential information (Burmeister et al., 2009; Munchrath and Hofmann 2010; Simões et al., 2012) for a number of Lamprologine cichlids (N. pulcher: D. Antunes et al., unpublished data; N. multifasciatus, Neolamprologus similis, $N$. brevis, L. ocellatus, L. sp. 'ornatipinnis zambia', Neolamprologus meeli, Telmatochromis temporalis 'dwarf morph': Lein et al., in preparation). These morphological atlases describe SDMN-associated nuclei within the brain and enable the comparison of volumes and neuronal densities of brain (sub-)structures and SDMN nuclei. In birds, neuronal density in the telencephalon has been shown to be a better predictor for cognitive performance as compared to brain size (Olkowicz et al., 2016). Consequently, quantifications on the cellular level in candidate areas of the brain (i.e. SDMN-associated nuclei) constitute an important step towards refinement beyond comparably coarse measurements of cognitive capacity, like (relative) brain size (e.g. van Staaden et al., 1994; Huber et al., 1997; Gonzalez-Voyer et al., 2009; Tsuboi et al., 2015) or volumes of larger brain areas such as the telencephalon or hypothalamus (e.g. in Pollen et al., 2007; Shumway, 2008; Gonzalez-Voyer and Kolm 2010). Indeed, the use of coarse neuroanatomy as an explanatory variable in understanding the function or evolution of the nervous system has come under criticism (Healy \& Rowe, 2007; Pollen \& Hofmann, 2008; Chittka et al., 2012).

Over the past decades, a handful of African cichlid species have emerged as model systems in the field of neuroethology, with numerous studies covering a wide array of different aspects including the mechanistic basis of behaviour (Astatotilapia burtoni: Greenwood et al., 2008; Maruska \& Fernald, 2018; O'Connell et al., 2011; Oreochromis mossambicus: Almeida et al., 2019; and N. pulcher: Taborsky et al., 2013; Kasper et al., 2018a; Kasper et al., 2018b). Leaning on this extensive body of knowledge, morphological brain atlases in shell-dwelling cichlids will facilitate studies into patterns of neural activity in response to social stimuli through quantification of immediate early gene (IEG) expression levels (e.g. transcription factors $c$-fos, egr- 1 ) in candidate regions of the SDMN. Such transcriptional profiling is a frequently used method in neuroethological studies involving fishes (Maruska et al., 2013; Desjardins et al., 2015; Teles et al., 2015; Teles et al., 2016; Roleira et al., 2017; Weitekamp \& Hofmann, 2017) and appears to increasingly corroborate the functional significance of SDMN nuclei in encoding social behaviour.

\section{Further advantages}

Field-based studies

A major strength of the Lamprologine system is that natural experiments in Lake Tanganyika are tractable, due to the small size of study animals, their small home ranges, and their site fidelity. Moreover, although access to Lake Tanganyika remains somewhat difficult, conditions at the Lake are highly conducive for underwater field work. Lake Tanganyika is one of the clearest bodies of freshwater in the world (Konings, 2015), and downwelling surface irradiance reaches the lake benthos in near-shore waters (Langenberg et al., 2002). This means that shell-dwelling cichlids, which typically inhabit depths of $\sim 10 \mathrm{~m}$, can be observed with relative ease in their natural habitat through SCUBA diving (Konings, 2015). Home ranges of the shell-dwelling cichlids species are often very small (e.g. N. multifasciatus or T. temporalis 'dwarf morph': approx. $30 \mathrm{~cm}$; Schradin and Lamprecht, 2002; EL personal obs.), and individuals usually restrict their movements to around and between those shells that lie within their own territory boundaries (e.g. Konings, 2015; Jordan et al., 2016; EL, personal obs.). This is of high practical utility, since it allows researchers to monitor the location and behaviour of multiple individuals simultaneously and continuously using either direct observation or by placing camera arrays. The latter method is particularly advantageous considering that, after prior installation of multi-camera arrays, individual fish can be observed in a minimally invasive way, and both their natural behaviour and the community species composition can be captured as accurately as possible (Widmer et al., 2019). Moreover, a strong site fidelity and the association of individuals with their "home shells", whose position can be manipulated, make it possible to manipulate distances among individuals in a group, modify available resources (Jordan et al., 2016), or even manipulate the shells themselves (Bose et al., 2020). These manipulations facilitate 
investigations into the potential of social plasticity in different species of shell-dwelling cichlids, allow examinations of the effect of early-life social environment on the acquisition of social competence (Fischer et al., 2015; Fischer et al., 2017b; Nyman et al., 2017), or assessments with respect to brain development (Fischer et al., 2015).

\section{Laboratory-based studies}

Small body sizes, short generation times (Koblmüller et al., 2008), the possibility to obtain large numbers of offspring under semi-natural laboratory settings, in combination with their unproblematic conservation status (Least concern in all cases, https://www. iucnredlist.org/), also allow for the establishment of sizable populations in laboratory conditions and therefore make shell-dwelling cichlids amenable for investigating e.g. neuroanatomy or cognitive performance with a high sampling throughput. By contrast, in other commonly investigated study systems like primates ethical or logistical considerations and resulting limited sample sizes often hamper the interpretability of the obtained data. A promising future avenue for studying social behaviour lies furthermore in the use of CRISPR gene editing and other genetic tools that allow to selectively manipulate candidate neural circuits involved in social interactions (Juntti, 2019), or the use of immersive virtual reality (VR) to experimentally control and alter an individual's perception of its social environment. The adaptation of these techniques in fish has been pioneered in zebrafish (Hruscha et al., 2013; Liu et al., 2018; Stowers et al., 2017) and has recently also been applied to cichlids (Juntti et al., 2016; Alward et al., 2020). Thus, an extension to non-traditional model systems like shell-dwelling cichlids seems not only realizable, but crucially, would also yield highly interesting new insights given their evolutionary divergence into distinct social strategies (Juntti, 2019).

\section{Outstanding challenges in the study of social and cognitive evolution}

To properly test the link between social and environmental conditions, cognitive, and brain evolution, it is essential to identify those factors of social living that affect the cognitive workload of an animal (Bergman
\& Beehner, 2015). As we have described above, one limitation comes where often large variation in ecology and geography among species being compared obscures any effects of differences in social systems. However, a second and perhaps more difficult problem comes where the classification of social complexity is inadequate, weakening subsequent comparisons to quantitative traits like brain morphology. Initial proxies for social complexity like average group size (Dunbar \& Shultz, 2007) have proven inadequate (e.g. Sandel et al., 2016), and more recent definitions have, for instance, emphasized the importance of differentiated relationships in stable social groups (Shultz \& Dunbar, 2006; Bergman \& Beehner, 2015), arguing that the necessary behavioural flexibility is cognitively challenging and therefore exerts a selective pressure driving cognitive evolution. Although this definitional refinement has gained traction in certain areas of social evolution research (e.g. in primatology: Fischer et al., 2017a; Kappeler 2019; Lukas \& Clutton-Brock, 2018; Ramos-Fernandez et al., 2018), likely due to constraints in the ability to resolve social structure in sufficient detail, many studies still rely on categorical or coarse quantitative classifications (e.g. Weisbecker et al., 2015; DeCasien et al., 2017; Fox et al., 2017; Ashton et al., 2018; Kverková et al., 2018). Similarly, in cichlid research, categorical classifications of sociality (based for instance on qualitative descriptions of mating system or parental care type; often from hobbyist publications) are still rather the norm than the exception. Cooperatively breeding species like $N$. pulcher or the shell-dweller $N$. multifasciatus are regularly attributed to be highly social, highly advanced social, or interchangeably, (highly) socially complex (e.g. Mileva et al., 2009; Pisanski et al., 2015; Jordan et al., 2016; Fischer et al., 2017b; Taborsky \& Wong, 2017). The justification for this label varies but has been connected, amongst others, to the existence of social stratification, individualized relationships, cooperation, and/or frequent social interactions (Pisanski et al., 2015; Reddon et al., 2016; Fischer et al., 2017b). While there is no doubt that $N$. pulcher experiences a complex social environment, it remains unclear which combination of social factors generates the complexity experienced by an individual, and how these factors combine and scale. This knowledge is essential for cross-species comparisons in which the robustness of the data and the conclusions that can be 
drawn from them strongly hinges on a fine resolution in the independent variable of interest.

As pointed out before elsewhere (e.g. Bergman \& Beehner, 2015), qualitative classifications are inherently problematic when applied to questions dealing with social and cognitive co-evolution, since their bearing on the cognitive load can generate ambiguous and contradictory predictions. For instance, it has been argued that monogamy is highly complex, since bond maintenance is thought to require an increased level of social acuity, deception (Schillaci 2006), and/or coordination abilities (Emery et al., 2007; Shultz \& Dunbar, 2007). On the other hand, the management of multiple relationships and more complex interactions in polygamous systems has been connected with high cognitive demands alike (e.g. Sawaguchi 1992). As for cooperative breeding, a recent study on sixteen nominally cooperatively and independently breeding Lamprologine species detected no positive correlation between cooperative breeding and increased brain size (Reddon et al., 2016), which might support the argument that it is not cooperative breeding per se that is cognitively challenging (Thornton \& McAuliffe, 2015).

A major limitation of any such species-level approaches is that a single species can often occur in monogamous or polygamous associations, depending on population, resource availability, location within a community, or time of year (see Table 2; Limberger, 1983; Heg \& Bachar, 2006; Desjardins et al., 2008; Smuts et al., 2008), making an either/or classification inherently problematic. Mating system-based classifications of social complexity are further complicated because often no clear distinction between social (i.e. inferred through behavioural observations) and genetic (i.e. based on parentage analysis) mating systems is being made (Sefc, 2011). This ambiguity, potentially resulting from definitional obscurities in combination with a general uncertainty concerning mating systems in Lamprologine cichlids (Taborsky et al., 2019), has recently sparked a controversy within the community (Tanaka et al., 2018; Dey et al., 2019), emphasizing the difficulties in using a mating systembased approach to the definition of social complexity.

An alternative, but equally problematic categorical division in Lamprologine cichlids is often made between grouping and non-grouping species, which may then be further broken down as (highly) social and less social (e.g. Hick et al., 2014; Balshine et al.,
2017). Although the required criterion of a link between sociality and cognitive load appears more straightforward in this scheme, grouping and nongrouping at best offer a similar proxy for social complexity as does group size, insofar as it describes a coarse number of (theoretical) interaction partners. Classifications such as grouping vs. non-grouping are further weakened by the difficulty in assigning animal group membership based on anthropocentric metrics of, e.g. distance. Although it is straightforward to define individuals as belonging to the same group if they are within some distance range of one another, this may not reflect the perceived sensory environment of the animals being studied (Jordan \& Ryan, 2015). The term "social" implies the occurrence of interactions between conspecific individuals, which can occur over large visual ranges (and in other modalities) than are easily captured by casual observations of physical proximity. As an example, the typical distances over which harems of $N$. multifasciatus interact is much smaller than the equivalent distance in L. ocellatus harems (Gordon and Bills 1999; Trillmich et al., 1999; Schradin \& Lamprecht, 2002; Jordan et al., 2016), and therefore it cannot be concluded that N. multifasciatus is grouping while L. ocellatus is not. Even if rigorously defined, these dichotomous classes leave no room for intermediate levels of organization, into which many species realistically naturally fall (Krause et al., 2002). N. pulcher, for instance, exhibits strong variation with regard to group sizes; although groups typically consist of 7-9 individuals on average, groups with only three and up to 38 individuals have been observed in the natural habitat (Balshine et al., 2001; Taborsky et al., 2005). Likewise, for $N$. multifasciatus, and T. temporalis 'dwarf morph', reports of group sizes range from 2 to 20 individuals (Kohler 1998; Schradin \& Lamprecht, 2002; Konings, 2015; Bose et al., in preparation) and 2 to 7 individuals (Takahashi et al., 2012), respectively.

A quantitative alternative is the representation of conspecific individuals in the form of social networks (Wey et al., 2008; Jordan et al., 2016). In social networks, each individual is represented as a node that can share a connection (= "edge") with another node based on a defined criterion, such as the presence of a behavioural, spatial, or temporal association between the two nodes (Croft et al., 2008). Social network theory (SNT) has previously been applied to different contexts in the study of Lamprologine cichlids (e.g. in 
the context of intra-group social dynamics: Hellmann \& Hamilton, 2019; in the context of extended phenotypes: Jordan et al., 2016; in the context of investigating dominance interactions and spatial associations: Dey et al., 2013; in the context of general reciprocity: van Doorn \& Taborsky, 2012; in the context of behavioural types: Schuerch et al., 2010). Despite these various cases of application, however, the utility of SNT for conceptualizing Lamprologine social complexity has not been realized. As an alternative to mating system- or proximityinfluenced classifications, a social network-based approach that seeks to approximate social complexity on the basis of interaction data in an objective, quantitative manner would allow to capture the social complexity in its multi-dimensional nature, arising as the simultaneous interplay of several contributing factors (e.g. the number of different interaction partners, the frequencies with which they interact, and the qualities of these interactions). In contrast to the term grouping, which may suffer from confounding effects of proximity, group cut-offs in social networks ("network boundaries") can be defined explicitly based on recorded interaction data (Laumann et al., 1989). On the other hand, and in contrast to classifications based on dichotomous categories, variations in group size and their suggested implication for social complexity (Groenewoud et al., 2016) are well quantified in a social network approach, in which any number of individuals (or even heterospecifics, which have been suggested to contribute to social complexity; Bshary et al., 2014) can theoretically be aggregated into the analytical unit.

Most crucially and contrary to broad or binary classifications schemes, a quantitative approach achieved through social network analysis (SNA) would afford a better resolution of the independent variable (i.e. social complexity) and therefore allow for a stronger test of the hypothesized positive relationship between social complexity and cognitive capacity. A previous study investigating different populations of $N$. pulcher found a considerable interpopulation variability in the size (i.e. number of individuals) and composition of social groups, resulting from local differences in predator abundance (Groenewoud et al., 2016). Not least because many shell-dwelling cichlids share sympatric patterns of distribution with $N$. pulcher (Konings, 2015), it is conceivable that analogous effects of variation in the risk environment also affect intraspecific variation in social complexity in shell-dwelling cichlids. Intraspecific variation in the social environment has recently been shown to affect the size of brain parts involved in socially relevant cognitive functions in the bluestreak cleaner wrasse Labroides dimidiatus (Triki et al., 2019). While such changes represent an insurmountable obstacle for binary classification schemes, variation on this scale can be addressed by means of SNA and thereby create additional refinement in the social dimension.

\section{Emerging quantitative approaches to address current limitations}

A reasonable criticism of social network approaches is that essential detail on behavioural interactions is lost in the compression of data to network edges. This problem can arise for two reasons. The first is that human observation skills are restricted to attending to one or two focal individuals at a given time (although see Pruitt \& Pinter-Wollman, 2015, for a study in which the personality and behaviour of 40 individuals were, allegedly, simultaneously recorded by a single observer). This restriction on human attention necessarily means that social networks must be constructed via successive focal sampling of individuals (e.g. Jordan et al., 2016), thereby reducing the amount of data collected per individual, especially under the time constraints induced by SCUBA-based field work. The second is that many different behaviours that are observed may be simplified to edges representing broader categories such as "aggressive interactions". This may be required because certain behaviours are rare and so creating networks on each behaviour result in overly sparse networks that cannot easily be analysed, and this problem is exacerbated when sampling times are short. As such, the potential for SNA to improve resolution of social structure and behaviour may be constrained by the ability to collect sufficient data. To date, this criticism has been justified, but modern approaches in behavioural ecology are well poised to overcome these constraints.

Recently, the explosion of computer vision and machine learning algorithms have made it possible to automatically track both the location and the posture of animals under controlled conditions (Brown \& De Bivort, 2018), and these techniques have even been 
extended to complex underwater environments including Lake Tanganyika (Francisco et al., 2020). With these technologies, which are based on training artificial neural networks to detect fish in natural scenes, it is increasingly feasible to record and subsequently automatically analyse the behaviour of anywhere from one to hundreds of animals in incredible detail. These high-throughput methodologies will revolutionize the field of behavioural ecology and are particularly applicable to Lacustrine underwater contexts. While it is easiest to work in clear water with animals that are easily distinguishable from their background, these approaches have already been proven effective under challenging environmental settings such as those posed by an aquatic environment like Lake Tanganyika, where conditions of low light, murky water, crypsis of the focal animal (e.g. in $T$. temporalis 'dwarf morph', Takahashi, 2019), occurrences of plankton blooms (Plisnier et al., 1999; Salonen et al., 1999), heterogeneous backgrounds, or backdrops with varying light incidence, occur at times. These automated tracking approaches can rapidly generate rich datasets on the identity, position, motion, and posture of all group members under observation, relying on consumer grade cameras (Francisco et al., 2020). As such, rich behavioural and association networks can be populated and analysed without the constraints imposed by human observation, even in the natural contexts in which individuals within a species or a population have evolved.

A second great opportunity presented by automated tracking is the ability to analyse behavioural states in great detail. In addition to the objective, quantitative metrics of social complexity achievable by tracking numerous individuals in real time, computational methods allow to objectively quantify and understand the behaviours themselves. These techniques, known broadly as "behavioural decomposition", have become generally available and are enjoying increasing popularity, allowing a transition from qualitative ethological description to quantitative analyses of behaviour (Berman et al., 2014). These approaches take time series data of animal postures and subdivide these into discrete clusters, either with supervised or unsupervised algorithms. Behavioural decomposition employs clustering with stochastical neighbour embedding and subsequently separates clusters into distinct states that can be interpreted as stereotypical behaviours (Berman et al., 2014; Klibaite et al., 2016).
Alternatively, machine learning algorithms can be trained on a small, annotated subset of the recorded time series to detect the annotated behaviours, massively increasing the quantity of behavioural data able to be gathered (Kabra et al., 2013). With these approaches, the scoring of behavioural traits becomes consistently repeatable across studies and species, while also reducing the potential for human subjectivity influencing data, which may lead to disagreement over categories or definitions of behaviour (Brown \& De Bivort, 2018). These approaches also have the potential to identify previously unrecognized behaviour, thus adding further resolution to behavioural analyses. Lamprologine cichlids are particularly well suited to these types of analyses, given their high site fidelity, small home ranges, and small elevation from the benthos, all of which allow relatively straightforward video recording and subsequent tracking. Together with the emerging field of animal linguistics that investigates syntax patterns and semantics in social behaviour, these approaches will allow to capture the true richness of interactions between these fascinating animals. Finally, the ability to produce aquarium hybrids, and to potentially observe such hybrids in the wild (Koblmüller et al., 2007) makes it conceivable to establish and study "intermediate" social phenotypes in this study system, which would eventually allow for an even more nuanced analysis of social complexity.

\section{Conclusion}

We argue that Tanganyikan shell-dwelling cichlids represent a unique system for assessing the effect of the social environment on brain/cognitive evolution. Currently used classifications of sociality or social complexity, however, fall short of capturing a relevant metric of the cognitive demands related to the social environment of individuals within differing groups, populations, or species. As an alternative, and in line with recent developments towards more meaningful appraisal of social complexity within the field of comparative cognition, we therefore propose a shift towards a social network-based approach in combination with emerging tracking technologies that strives to incorporate the multi-dimensional nature of social complexity in an information theoretical framework. In this way, we can bring cichlids to the 
forefront of research into the social mechanisms leading to brain and behavioural evolution.

Investigating the proposed link between social and cognitive evolution is contingent upon a holistic understanding of the respective studied system. Using Tanganyikan shell-dwelling cichlids in such a framework will consequently require filling still existing gaps and uncertainties, particularly with regard to the ecology of a few, currently understudied, species. These data will be especially pertinent to clarify valid reservations with regard to the extent of actual ecological variation among shell-dwelling species. With more information on hand, shell-dwelling cichlids have then the potential to provide a truly unique model system to address the predictions made by the social brain hypothesis and related conceptual frameworks.

Acknowledgements The authors would like to thank the organizing committee of the Cichlid Science 2019 conference held in Madrid, Spain, as well as all members of the Jordan lab for their constructive comments and suggestions. We thank Heinz Büscher and Ad Konings for their immensely valuable feedback regarding cichlid behavioural ecology, and Fabrizia Ronco and Walter Salzburger for providing information on the trophic signatures of Tanganyikan shell-dwelling cichlids. We furthermore thank Kristina Sefc and two anonymous reviewers for their helpful comments and insightful suggestions for improvements on earlier versions of this manuscript.

Author contributions Etienne Lein and Alex Jordan conceptualized the article. Etienne Lein performed the literature search and together with Alex Jordan drafted and revised the manuscript critically. Both authors approved the manuscript in its final version for submission.

Funding Open Access funding enabled and organized by Projekt DEAL. This work was funded by the Deutsche Forschungsgemeinschaft (DFG, German research Foundation) under Germany's Excellence Strategy - EXC 2117 - 422037984 and by the Max Planck Institute of Animal Behavior.

\section{Compliance with ethical standards}

Conflict of interest The authors declare no conflict of interest.

Ethical approval All behavioural observations were conducted with the approval of the Department of Fisheries, Ministry of Agriculture and Livestock, Zambia.

Open Access This article is licensed under a Creative Commons Attribution 4.0 International License, which permits use, sharing, adaptation, distribution and reproduction in any medium or format, as long as you give appropriate credit to the original author(s) and the source, provide a link to the Creative Commons licence, and indicate if changes were made. The images or other third party material in this article are included in the article's Creative Commons licence, unless indicated otherwise in a credit line to the material. If material is not included in the article's Creative Commons licence and your intended use is not permitted by statutory regulation or exceeds the permitted use, you will need to obtain permission directly from the copyright holder. To view a copy of this licence, visit http://creativecommons.org/licenses/by/4.0/.

\section{References}

Alcazar, R. M., A. T. Hilliard, L. Becker, M. Bernaba \& R. D. Fernald, 2014. Brains over brawn: experience overcomes a size disadvantage in fish social hierarchies. Journal of Experimental Biology 217(9): 1462-1468.

Alexander, R. D., 1974. The evolution of social behavior. Annual Review of Ecology and Systematics 5(1): 325-383.

Almeida, O., A. S. Félix, G. A. Oliveira, J. S. Lopes \& R. F. Oliveira, 2019. Fighting assessment triggers rapid changes in activity of the brain social decision-making network of cichlid fish. Frontiers in Behavioral Neuroscience 13: 229.

Alward, B. A., V. Laud, C. J. Skalnik, R. A. York, S. A. Juntti \& R. D. Fernald, 2020. Modular genetic control of social status in a cichlid fish. bioRxiv.

Arnold, C. \& B. Taborsky, 2010. Social experience in early ontogeny has lasting effects on social skills in cooperatively breeding cichlids. Animal Behaviour 79(3): 621-630.

Aronson, L. R., 1949. An analysis of reproductive behavior in the mouthbreeding cichlid fish, Tilapia macrocephala (Bleeker). Zoologica, New York.

Ashton, B. J., A. R. Ridley, E. K. Edwards \& A. Thornton, 2018. Cognitive performance is linked to group size and affects fitness in Australian magpies. Nature 554(7692): 364-367.

Baerends, G. P. \& J. Baerends-van Roon, 1950. An introduction to the study of the ethology of the cichlid fishes. Behaviour Supplement II I: 243.

Balshine-Earn, S. \& A. Lotem, 1998. Individual recognition in a cooperatively breeding cichlid: evidence from video playback experiments. Behaviour 135(3): 369-386.

Balshine, S., B. Leach, F. Neat, H. Reid, M. Taborsky \& N. Werner, 2001. Correlates of group size in a cooperatively breeding cichlid fish (Neolamprologus pulcher). Behavioral Ecology and Sociobiology 50(2): 134-140.

Balshine, S., M. Y. Wong \& A. R. Reddon, 2017. Social motivation and conflict resolution tactics as potential building blocks of sociality in cichlid fishes. Behavioural Processes 141: 152-160.

Barton, R. A., 1996. Neocortex size and behavioural ecology in primates. Proceedings of the Royal Society of London, Series B: Biological Sciences 263(1367): 173-177.

Beauchamp, G. \& E. Fernández-Juricic, 2004. Is there a relationship between forebrain size and group size in birds? Evolutionary Ecology Research 6(6): 833-842.

Bergman, T. J. \& J. C. Beehner, 2015. Measuring social complexity. Animal Behaviour 103: 203-209.

Berman, G. J., D. M. Choi, W. Bialek \& J. W. Shaevitz, 2014. Mapping the stereotyped behaviour of freely moving fruit 
flies. Journal of the Royal Society, Interface 11(99): 20140672.

Bills, I. \& A. Ribbink, 1997. Description of Lamprologus laparogramma sp. nov., and rediagnosis of Lamprologus signatus Poll 1956 and Lamprologus kungweensis Poll 1952, with notes on their ecology and behaviour (Teleostei: Cichlidae). South African Journal of Science 93: 555-564.

Bills, I. R., 1996. Eco-ethology of shell-dwelling cichlids in Lake Tanganyika. Rhodes University Grahamstown, South Africa.

Bolnick, D. I., R. Svanbäck, M. S. Araújo \& L. Persson, 2007. Comparative support for the niche variation hypothesis that more generalized populations also are more heterogeneous. Proceedings of the National Academy of Sciences 104(24): 10075-10079.

Booth, D. J., 1995. Juvenile groups in a coral-reef damselfish: density-dependent effects on individual fitness and population demography. Ecology 76(1): 91-106.

Bose, A. P., J. Windorfer, F. Ronco, A. Boehm, A. Indermaur, W. Salzburger \& A. L. Jordan, 2020. Structural manipulations of a shelter resource reveal underlying preference functions in a shell-dwelling cichlid fish. bioRxiv.

Brandtmann, G., 2002. Intrasexuelle Konflikte bei weiblichen Schneckencichliden Lamprologus ocellatus.

Brown, A. E. \& B. De Bivort, 2018. Ethology as a physical science. Nature Physics 14(7): 653-657.

Brown, C., K. Laland \& J. Krause, 2011. Fish cognition and behavior, Vol. 21. Wiley, New York.

Bruintjes, R. \& M. Taborsky, 2011. Size-dependent task specialization in a cooperative cichlid in response to experimental variation of demand. Animal Behaviour 81(2): 387-394.

Bshary, R. \& C. Brown, 2014. Fish cognition. Current Biology 24(19): R947-R950.

Bshary, R., S. Gingins \& A. L. Vail, 2014. Social cognition in fishes. Trends in Cognitive Sciences 18(9): 465-471.

Bshary, R., W. Wickler \& H. Fricke, 2002. Fish cognition: a primate's eye view. Animal Cognition 5(1): 1-13.

Burmeister, S. S., R. G. Munshi \& R. D. Fernald, 2009. Cytoarchitecture of a cichlid fish telencephalon. Brain, Behavior and Evolution 74(2): 110-120.

Büscher, H. H., 1991. Neue Schneckencichliden aus dem Tanganjikasee: Lamprologus meleagris $\mathrm{n}$. sp. und L. speciosus n. sp. (Cichidae, Lamprologini). Die Aquarien- und Terrarienzeitschrift (DATZ) 44: 374-382.

Büscher, H. H., 1992a. Neolamprologus leleoupi und N. caudopunctatus. Die Aquarien- und Terrarienzeitschrift (DATZ) 45: 39-44.

Büscher, H. H., 1992b. Ein neuer Cichlide aus dem Tanganjikasee: Neolamprologus similis n. sp. Die Aquarien- und Terrarienzeitschrift (DATZ) 45: 520-525.

Büscher, H. H., 1998. Eigenheim aus zweiter Hand: Buntbarsche in Schneckenhäusern. Die Aquarien-und Terrarienzeitschrift (DATZ) special issue Tanganjikasee 6:5159.

Chittka, L., S. J. Rossiter, P. Skorupski \& C. Fernando, 2012. What is comparable in comparative cognition? Philosophical Transactions of the Royal Society B: Biological Sciences 367(1603): 2677-2685.

Chojnacka, D., K. Isler, J. J. Barski \& R. Bshary, 2015. Relative brain and brain part sizes provide only limited evidence that machiavellian behaviour in cleaner wrasse is cognitively demanding. PloS ONE 10: 8.

Clabaut, C., P. M. Bunje, W. Salzburger \& A. Meyer, 2007. Geometric morphometric analyses provide evidence for the adaptive character of the Tanganyikan cichlid fish radiations. Evolution 61(3): 560-578.

Clutton-Brock, T. H. \& P. H. Harvey, 1980. Primates, brains and ecology. Journal of Zoology 190(3): 309-323.

Cohen, A. S. \& C. Thouin, 1987. Nearshore carbonate deposits in Lake Tanganyika. Geology 15(5): 414-418.

Coolen, I., 2002. Increasing foraging group size increases scrounger use and reduces searching efficiency in nutmeg mannikins (Lonchura punctulata). Behavioral Ecology and Sociobiology 52(3): 232-238.

Croft, D. P., R. James \& J. Krause, 2008. Exploring animal social networks. Princeton University Press, Princeton.

Davies, N. B., J. R. Krebs \& S. A. West, 2012. An introduction to behavioural ecology. Wiley, New York.

De Wever, A., K. Muylaert, K. Van der Gucht, S. Pirlot, C. Cocquyt, J.-P. Descy, P.-D. Plisnier \& W. Vyverman, 2005. Bacterial community composition in Lake Tanganyika: vertical and horizontal heterogeneity. Applied and Environmental Microbiology 71(9): 5029-5037.

Deaner, R. O., C. L. Nunn \& C. P. van Schaik, 2000. Comparative tests of primate cognition: different scaling methods produce different results. Brain, Behavior and Evolution 55(1): 44-52.

DeCasien, A. R., S. A. Williams \& J. P. Higham, 2017. Primate brain size is predicted by diet but not sociality. Nature Ecology \& Evolution 1(5): 1-7.

Desjardins, J. K., L. Becker \& R. D. Fernald, 2015. The effect of observers on behavior and the brain during aggressive encounters. Behavioural Brain Research 292: 174-183.

Desjardins, J. K., J. L. Fitzpatrick, K. A. Stiver, G. J. Van der Kraak \& S. Balshine, 2008. Costs and benefits of polygyny in the cichlid Neolamprologus pulcher. Animal Behaviour 75(5): 1771-1779.

Desjardins, J. K., H. A. Hofmann \& R. D. Fernald, 2012. Social context influences aggressive and courtship behavior in a cichlid fish. PLoS ONE 7: 7.

Dey, C. J., C. M. O'Connor, H. Wilkinson, S. Shultz, S. Balshine, et al., 2019. Confounding social and mating systems predictably lead to biased results when examining the evolution of cooperative breeding in cichlids: a response to Tanaka. Ethology 125(6): 409-414.

Dey, C. J., A. R. Reddon, C. M. O'Connor \& S. Balshine, 2013. Network structure is related to social conflict in a cooperatively breeding fish. Animal Behaviour 85(2): 395-402.

Dunbar, R. \& S. Shultz, 2017. Why are there so many explanations for primate brain evolution? Philosophical Transactions of the Royal Society B: Biological Sciences 372(1727): 20160244.

Dunbar, R. I., 1992. Neocortex size as a constraint on group size in primates. Journal of Human Evolution 22(6): 469-493.

Dunbar, R. I., 1998. The social brain hypothesis. Evolutionary Anthropology: Issues, News, and Reviews: Issues, News, and Reviews 6(5): 178-190.

Dunbar, R. I. \& S. Shultz, 2007. Evolution in the social brain. Science 317(5843): 1344-1347.

El-Zarka, S., 1956. Breeding behavior of the Egyptian cichlid fish, Tilapia zilli. Copeia 1956(2): 112-113. 
Emery, N. J., A. M. Seed, A. M. Von Bayern \& N. S. Clayton, 2007. Cognitive adaptations of social bonding in birds. Philosophical Transactions of the Royal Society B: Biological Sciences 362(1480): 489-505.

Fischer, J., M. S. Farnworth, H. Sennhenn-Reulen \& K. Hammerschmidt, 2017a. Quantifying social complexity. Animal Behaviour 130: 57-66.

Fischer, S., M. Bessert-Nettelbeck, A. Kotrschal \& B. Taborsky, 2015. Rearing-group size determines social competence and brain structure in a cooperatively breeding cichlid. The American Naturalist 186(1): 123-140.

Fischer, S., L. Bohn, E. Oberhummer, C. Nyman \& B. Taborsky, $2017 \mathrm{~b}$. Divergence of developmental trajectories is triggered interactively by early social and ecological experience in a cooperative breeder. Proceedings of the National Academy of Sciences 114(44): E9300-E9307.

Fox, K. C., M. Muthukrishna \& S. Shultz, 2017. The social and cultural roots of whale and dolphin brains. Nature Ecology \& Evolution 1(11): 1699-1705.

Francisco, F. A., P. Nührenberg \& A. Jordan, 2020. High-resolution, non-invasive animal tracking and reconstruction of local environment in aquatic ecosystems. Movement Ecology 8(1): 1-12.

Frostman, P. \& P. T. Sherman, 2004. Behavioral response to familiar and unfamiliar neighbors in a territorial cichlid, Neolamprologus pulcher. Ichthyological Research 51(3): 283-285.

Fryer, G. \& T. D. Iles, 1972. Cichlid fishes of the great lakes of Africa.

Gashagaza, M. \& M. Nagoshi, 1986. Comparative study on the food habits of six species of Lamprologus (Osteichthyes: Cichlidae). African Study Monographs 6: 37-44.

Gashagaza, M. M., 1988. Feeding activity of a Tanganyikan cichlid fish Lamprologus brichardi. African Study Monographs 9: 1-9.

Gashagaza, M., K. Nakaya \& T. Sato, 1995. Taxonomy of smallsized cichlid fishes in the shell-bed area of Lake Tanganyika. Japanese Journal of Ichthyology 42(3-4): 291-302.

Gibson, K., 1986. Cognition, brain size and the extraction of embedded food resources. Primate ontogeny, cognition and social behaviour.

Gingins, S. \& R. Bshary, 2016. The cleaner wrasse outperforms other labrids in ecologically relevant contexts, but not in spatial discrimination. Animal Behaviour 115: 145-155.

Gittleman, J. L., 1986. Carnivore brain size, behavioral ecology, and phylogeny. Journal of Mammalogy 67(1): 23-36.

Goldschmidt, T., 1998. Darwin's dreampond: drama in Lake Victoria. MIT Press, New York.

Gonzalez-Voyer, A. \& N. Kolm, 2010. Sex, ecology and the brain: evolutionary correlates of brain structure volumes in Tanganyikan cichlids. PLoS ONE. https://doi.org/10.1371/ journal.pone.0014355.

Gonzalez-Voyer, A., S. Winberg \& N. Kolm, 2009. Social fishes and single mothers: brain evolution in African cichlids. Proceedings of the Royal Society B: Biological Sciences 276(1654): 161-167.

Gonzalez-Voyer, A., J. L. Fitzpatrick \& N. Kolm, 2008. Sexual selection determines parental care patterns in cichlid fishes. Evolution: International Journal of Organic Evolution 62(8): 2015-2026.
Goodson, J. L., 2005. The vertebrate social behavior network: evolutionary themes and variations. Hormones and Behavior 48(1): 11-22.

Goodson, J. L. \& M. A. Kingsbury, 2013. What's in a name? Considerations of homologies and nomenclature for vertebrate social behavior networks. Hormones and Behavior 64(1): 103-112.

Gordon, A. K. \& I. R. Bills, 1999. Aspects of the feeding and reproductive biology of the Lake Tanganyikan cichlid, Lamprologus ornatipinnis (Pisces, Cichlidae). Environmental Biology of Fishes 55(4): 431-441.

Grafen, A. \& R. Sibly, 1978. A model of mate desertion. Animal Behaviour 26: 645-652.

Greenwood, A. K., A. R. Wark, R. D. Fernald \& H. A. Hofmann, 2008. Expression of arginine vasotocin in distinct preoptic regions is associated with dominant and subordinate behaviour in an African cichlid fish. Proceedings of the Royal Society B: Biological Sciences 275(1649): 2393-2402.

Groenewoud, F., J. G. Frommen, D. Josi, H. Tanaka, A. Jungwirth \& M. Taborsky, 2016. Predation risk drives social complexity in cooperative breeders. Proceedings of the National Academy of Sciences 113(15): 4104-4109.

Grosenick, L., T. S. Clement \& R. D. Fernald, 2007. Fish can infer social rank by observation alone. Nature 445(7126): 429-432.

Gutiérrez, J. L., C. G. Jones, D. L. Strayer \& O. O. Iribarne, 2003. Mollusks as ecosystem engineers: the role of shell production in aquatic habitats. Oikos 101(1): 79-90.

Healy, S. D. \& C. Rowe, 2007. A critique of comparative studies of brain size. Proceedings of the Royal Society B: Biological Sciences 274(1609): 453-464.

Heg, D. \& Z. Bachar, 2006. Cooperative breeding in the Lake Tanganyika cichlid Julidochromis ornatus. Environmental Biology of Fishes 76(2-4): 265-281.

Hellmann, J. K. \& I. M. Hamilton, 2019. Intragroup social dynamics vary with the presence of neighbors in a cooperatively breeding fish. Current Zoology 65(1): 21-31.

Hert, E., 1985. Individual recognition of helpers by the breeders in the cichlid fish Lamprologus brichardi (Poll, 1974). Zeitschrift für Tierpsychologie 68(4): 313-325.

Hick, K., A. R. Reddon, C. M. O'Connor \& S. Balshine, 2014. Strategic and tactical fighting decisions in cichlid fishes with divergent social systems. Behaviour 151(1): 47-71.

Hori, M., 1983. Feeding ecology of thirteen species of Lamprologus (Teleostei; Cichlidae) coexisting at a rocky shore of Lake Tanganyika. Physiology and Ecology Japan 20: 129-149.

Hotta, T., L. A. Jordan, T. Takeyama \& M. Kohda, 2015a. Order effects in transitive inference: does the presentation order of social information affect transitive inference in social animals? Frontiers in Ecology and Evolution 3: 59.

Hotta, T., S. Satoh, N. Kosaka \& M. Kohda, 2017. Face recognition in the Tanganyikan cichlid Julidochromis transcriptus. Animal Behaviour 127: 1-5.

Hotta, T., T. Takeyama, D. Heg, S. Awata, L. A. Jordan \& M. Kohda, 2015b. The use of multiple sources of social information in contest behavior: testing the social cognitive abilities of a cichlid fish. Frontiers in Ecology and Evolution 3: 85 . 
Hotta, T., T. Takeyama, L. A. Jordan \& M. Kohda, 2014. Duration of memory of dominance relationships in a group living cichlid. Naturwissenschaften 101(9): 745-751.

Hruscha, A., P. Krawitz, A. Rechenberg, V. Heinrich, J. Hecht, C. Haass \& B. Schmid, 2013. Efficient CRISPR/Cas9 genome editing with low off-target effects in zebrafish. Development 140(24): 4982-4987.

Huber, R., M. J. van Staaden, L. S. Kaufman \& K. F. Liem, 1997. Microhabitat use, trophic patterns, and the evolution of brain structure in African cichlids. Brain, Behavior and Evolution 50(3): 167-182.

Humphrey, N. K., 1976. The social function of intellect Growing points in ethology. Cambridge University Press, Cambridge: $303-317$.

Ito, M. H., M. Yamaguchi \& N. Kutsukake, 2018. Redirected aggression as a conflict management tactic in the social cichlid fish Julidochromis regani. Proceedings of the Royal Society B: Biological Sciences 285(1871): 20172681.

Jolly, A., 1966. Lemur social behavior and primate intelligence. Science 153(3735): 501-506.

Jordan, A., B. Taborsky \& M. Taborsky, 2020. Cichlids as a model system for studying social behaviour and evolution. In Abate, M. E. N. (ed.), The Behaviour, Ecology, and Evolution of cichlid fishes: a Contemporary Modern Synthesis. Springer Academic, Berlin.

Jordan, L. A. \& R. C. Brooks, 2012. Recent social history alters male courtship preferences. Evolution: International Journal of Organic Evolution 66(1): 280-287.

Jordan, L. A., H. Kokko \& M. Kasumovic, 2014. Reproductive foragers: male spiders choose mates by selecting among competitive environments. The American Naturalist 183(5): 638-649.

Jordan, L. A., S. M. Maguire, H. A. Hofmann \& M. Kohda, 2016. The social and ecological costs of an 'over-extended' phenotype. Proceedings of the Royal Society B: Biological Sciences 283(1822): 20152359.

Jordan, L. A. \& M. J. Ryan, 2015. The sensory ecology of adaptive landscapes. Biology Letters 11(5): 20141054.

Juntti, S., 2019. The future of gene-guided neuroscience research in non-traditional model organisms. Brain, Behavior and Evolution 93(2-3): 108-121.

Juntti, S. A., A. T. Hilliard, K. R. Kent, A. Kumar, A. Nguyen, M. A. Jimenez, J. L. Loveland, P. Mourrain \& R. D. Fernald, 2016. A neural basis for control of cichlid female reproductive behavior by prostaglandin F2 $\alpha$. Current Biology 26(7): 943-949.

Kabadayi, C., L. A. Taylor, A. M. von Bayern \& M. Osvath, 2016. Ravens, New Caledonian crows and jackdaws parallel great apes in motor self-regulation despite smaller brains. Royal Society Open Science 3(4): 160104.

Kabra, M., A. A. Robie, M. Rivera-Alba, S. Branson \& K. Branson, 2013. JAABA: interactive machine learning for automatic annotation of animal behavior. Nature Methods 10(1): 64.

Kappeler, P. M., 2019. A framework for studying social complexity. Behavioral Ecology and Sociobiology 73(1): 13.

Karlsson, M. \& M. Karlsson, 2020. Neolamprologus sp. "Mwila" eine neue Schneckencichlidenart von Mwila Island im Kipili-Archipel und die Wahrheit über den weniger bekannten N. sp. Eseki. DCG Informationen 51(1): 2-14.
Kasper, C., M. Colombo, N. Aubin-Horth \& B. Taborsky, 2018a. Brain activation patterns following a cooperation opportunity in a highly social cichlid fish. Physiology \& Behavior 195: 37-47.

Kasper, C., F. O. Hebert, N. Aubin-Horth \& B. Taborsky, 2018 b. Divergent brain gene expression profiles between alternative behavioural helper types in a cooperative breeder. Molecular Ecology 27(20): 4136-4151.

Klibaite, U., G. J. Berman, J. Cande, D. L. Stern \& J. W. Shaevitz, 2016. An Unsupervised Method for Quantifying the Behavior of Interacting Individuals. arXiv preprint. arXiv: 160909345.

Koblmüller, S., N. Duftner, K. M. Sefc, M. Aibara, M. Stipacek, M. Blanc, B. Egger \& C. Sturmbauer, 2007. Reticulate phylogeny of gastropod-shell-breeding cichlids from Lake Tanganyika: the result of repeated introgressive hybridization. BMC Evolutionary Biology 7(1): 7.

Koblmüller, S., K. M. Sefc \& C. Sturmbauer, 2008. The Lake Tanganyika cichlid species assemblage: recent advances in molecular phylogenetics. Hydrobiologia 615(1): 5.

Kocher, T. D., 2004. Adaptive evolution and explosive speciation: the cichlid fish model. Nature Reviews Genetics 5(4): 288-298.

Kohda, M., L. A. Jordan, T. Hotta, N. Kosaka, K. Karino, H. Tanaka, M. Taniyama \& T. Takeyama, 2015. Facial recognition in a group-living cichlid fish. PLoS ONE 10(11): e0142552.

Kohler, U., 1998. Zur Struktur und Evolution des Sozialsystems von Neolamprologus multifasciatus (Cichlidae, Pisces), dem kleinsten Schneckenbuntbarsch des Tanganjikasees. Shaker.

Konings, A., 1998. Tanganyika cichlids in their natural habitat. Cichlid Press, El Paso.

Konings, A., 2015. Tanganyika Cichlids in their natural habitat, 3rd ed. Cichlid Press, El Paso.

Kornfield, I. \& P. F. Smith, 2000. African cichlid fishes: model systems for evolutionary biology. Annual Review of Ecology and Systematics 31(1): 163-196.

Kotrschal, A., B. Rogell, A. A. Maklakov \& N. Kolm, 2012. Sex-specific plasticity in brain morphology depends on social environment of the guppy. Poecilia reticulata. Behavioral Ecology and Sociobiology 66(11): 1485-1492.

Krause, J., G. D. Ruxton, G. D. Ruxton \& I. G. Ruxton, 2002. Living in groups. Oxford University Press, Oxford.

Krebs, J. R., 1974. Colonial nesting and social feeding as strategies for exploiting food resources in the Great Blue Heron (Ardea herodias). Behaviour 51(1-2): 99-134.

Kverková, K., T. Bělíková, S. Olkowicz, Z. Pavelková, M. J. O'Riain, R. Šumbera, H. Burda, N. C. Bennett \& P. Němec, 2018. Sociality does not drive the evolution of large brains in eusocial African mole-rats. Scientific Reports 8(1): 1-14.

Langenberg, V., L. Mwape, K. Tshibangu, J.-M. Tumba, A. Koelmans, R. Roijackers, K. Salonen, J. Sarvala \& H. Mölsä, 2002. Comparison of thermal stratification, light attenuation, and chlorophyll-a dynamics between the ends of Lake Tanganyika. Aquatic Ecosystem Health \& Management 5(3): 255-265.

Laumann, E. O., P. V. Marsden \& D. Prensky, 1989. The boundary specification problem in network analysis. Research methods in social network analysis 61: 87. 
Lihoreau, M., T. Latty \& L. Chittka, 2012. An exploration of the social brain hypothesis in insects. Frontiers in Physiology 3: 442.

Lima, S. L., 1995. Back to the basics of anti-predatory vigilance: the group-size effect. Animal Behaviour 49(1): 11-20.

Lima, S. L. \& P. A. Bednekoff, 1999. Back to the basics of antipredatory vigilance: can nonvigilant animals detect attack? Animal Behaviour 58(3): 537-543.

Limberger, D., 1983. Pairs and Harems in a Cichlid Fish, Lamprologus brichardi 1 . Zeitschrift für Tierpsychologie 62(2): 115-144.

Liu, C.-X., C.-Y. Li, C.-C. Hu, Y. Wang, J. Lin, Y.-H. Jiang, Q. Li \& X. Xu, 2018. CRISPR/Cas9-induced shank3b mutant zebrafish display autism-like behaviors. Molecular Autism 9(1): 23.

Logan, C. J., S. Avin, N. Boogert, A. Buskell, F. R. Cross, A. Currie, S. Jelbert, D. Lukas, R. Mares \& A. F. Navarrete, 2018. Beyond brain size: uncovering the neural correlates of behavioral and cognitive specialization.

Lubin, Y. \& T. Bilde, 2007. The evolution of sociality in spiders. Advances in the Study of Behavior 37: 83-145.

Lukas, D. \& T. Clutton-Brock, 2018. Social complexity and kinship in animal societies. Ecology Letters 21(8): 1129-1134.

Maciejewski, M. F., C. Jiang, Y. E. Stuart \& D. I. Bolnick, 2020. Microhabitat contributes to microgeographic divergence in threespine stickleback. Evolution. https://doi.org/10.1111/ evo.13942.

MacLean, E. L., L. J. Matthews, B. A. Hare, C. L. Nunn, R. C. Anderson, F. Aureli, E. M. Brannon, J. Call, C. M. Drea \& N. J. Emery, 2012. How does cognition evolve? Phylogenetic comparative psychology. Animal cognition 15(2): 223-238.

Martinez, F. A. \& E. A. Marschall, 1999. A dynamic model of group-size choice in the coral reef fish Dascyllus albisella. Behavioral Ecology 10(5): 572-577.

Maruska, K. P. \& R. D. Fernald, 2018. Astatotilapia burtoni: a model system for analyzing the neurobiology of behavior. ACS Chemical Neuroscience 9(8): 1951-1962.

Maruska, K. P., A. Zhang, A. Neboori \& R. D. Fernald, 2013. Social opportunity causes rapid transcriptional changes in the social behaviour network of the brain in an African cichlid fish. Journal of Neuroendocrinology 25(2): 145-157.

Meyer, A., 1993. Phylogenetic relationships and evolutionary processes in East African cichlids. Trends in Ecology \& Evolution 8(8): 279-284.

Mileva, V. R., J. L. Fitzpatrick, S. Marsh-Rollo, K. M. Gilmour, C. M. Wood \& S. Balshine, 2009. The stress response of the highly social African cichlid Neolamprologus pulcher. Physiological and Biochemical Zoology 82(6): 720-729.

Mitchell, J. S., S. W. Ocana \& M. Taborsky, 2014. Male and female shell-brooding cichlids prefer different shell characteristics. Animal Behaviour 98: 131-137.

Morgan, M. J., 1988. The influence of hunger, shoal size and predator presence on foraging in bluntnose minnows. Animal Behaviour 36(5): 1317-1322.

Munchrath, L. A. \& H. A. Hofmann, 2010. Distribution of sex steroid hormone receptors in the brain of an African cichlid fish, Astatotilapia burtoni. Journal of Comparative Neurology 518(16): 3302-3326.
Newman, S. W., 1999. The medial extended amygdala in male reproductive behavior a node in the mammalian social behavior network. Annals of the New York Academy of Sciences 877(1): 242-257.

Noble, G. K., 1937. Effect of lesions of the corpus striatum on the brooding behavior of cichlid fishes. The Anatomical Record 70: 1 .

Noble, G. K. \& B. Curtis, 1939. The social behavior of the jewel fish, Hemichromis bimaculatus Gill. Bulletin of the Amarican Museum of Natural History 76: 1.

Nyman, C., S. Fischer, N. Aubin-Horth \& B. Taborsky, 2017. Effect of the early social environment on behavioural and genomic responses to a social challenge in a cooperatively breeding vertebrate. Molecular Ecology 26(12): 3186-3203.

Ochi, H. \& Y. Yanagisawa, 1999. Sand-transfer behavior outside the nest by guarding parents of the Tanganyikan cichlid, Neolamprologus caudopunctatus. Ichthyological Research 46(4): 419-422.

O'Connell, L. A., M. R. Fontenot \& H. A. Hofmann, 2011. Characterization of the dopaminergic system in the brain of an African cichlid fish, Astatotilapia burtoni. Journal of Comparative Neurology 519(1): 75-92.

O'Connell, L. A. \& H. A. Hofmann, 2011. The vertebrate mesolimbic reward system and social behavior network: a comparative synthesis. Journal of Comparative Neurology 519(18): 3599-3639.

O'Connell, L. A. \& H. A. Hofmann, 2012. Evolution of a vertebrate social decision-making network. Science 336(6085): 1154-1157.

Oh, K. P. \& A. V. Badyaev, 2010. Structure of social networks in a passerine bird: consequences for sexual selection and the evolution of mating strategies. The American Naturalist 176(3): E80-E89.

Oliveira, R. F., 2013. Mind the fish: zebrafish as a model in cognitive social neuroscience. Frontiers in Neural Circuits 7: 131.

Olkowicz, S., M. Kocourek, R. K. Lučan, M. Porteš, W. T. Fitch, S. Herculano-Houzel \& P. Němec, 2016. Birds have primate-like numbers of neurons in the forebrain. Proceedings of the National Academy of Sciences 113(26): 7255-7260.

Ota, K., M. Aibara, M. Morita, S. Awata, M. Hori \& M. Kohda, 2012. Alternative reproductive tactics in the shell-brooding Lake Tanganyika cichlid Neolamprologus brevis. International Journal of Evolutionary Biology. https://doi.org/ $10.1155 / 2012 / 193235$.

Ota, K. \& M. Kohda, 2006a. Description of alternative male reproductive tactics in a shell-brooding cichlid, Telmatochromis vittatus, Lake Tanganyika. Journal of Ethology 24(1): 9-15.

Ota, K. \& M. Kohda, 2006b. Nest use by territorial males in a shell-brooding cichlid: the effect of reproductive parasitism. Journal of Ethology 24(1): 91-95.

Parker, G. A., 1974. Courtship persistence and female-guarding as male time investment strategies. Behaviour 48(1-4): 157-183.

Parker, S. T. \& K. R. Gibson, 1977. Object manipulation, tool use and sensorimotor intelligence as feeding adaptations in Cebus monkeys and great apes. Journal of Human Evolution 6(7): 623-641. 
Pisanski, K., S. E. Marsh-Rollo \& S. Balshine, 2015. Courting and fighting quietly: a lack of acoustic signals in a cooperative Tanganyikan cichlid fish. Hydrobiologia 748(1): 87-97.

Pitcher, T., A. Magurran \& I. Winfield, 1982. Fish in larger shoals find food faster. Behavioral Ecology and Sociobiology 10(2): 149-151.

Pitcher, T. J., 1986. Functions of shoaling behaviour in teleosts: the behaviour of teleost fishes. Springer, Berlin: 294-337.

Plisnier, P.-D., D. Chitamwebwa, L. Mwape, K. Tshibangu, V. Langenberg \& E. Coenen, 1999. Limnological annual cycle inferred from physical-chemical fluctuations at three stations of Lake Tanganyika From limnology to fisheries: Lake Tanganyika and other large lakes. Springer, Berlin: 45-58.

Poll, M., 1956. Poissons Cichlidae. Resultats scientifique. Exploration hydrobiologique du Lac Tanganika. Institut Royal des Sciences Naturelles Belgique 3(5): 1-619.

Pollen, A. A., A. P. Dobberfuhl, J. Scace, M. M. Igulu, S. C. Renn, C. A. Shumway \& H. A. Hofmann, 2007. Environmental complexity and social organization sculpt the brain in Lake Tanganyikan cichlid fish. Brain, Behavior and Evolution 70(1): 21-39.

Pollen, A. A. \& H. A. Hofmann, 2008. Beyond neuroanatomy: novel approaches to studying brain evolution. Brain, Behavior and Evolution 72(2): 145-158.

Pruitt, J. N. \& N. Pinter-Wollman, 2015. The legacy effects of keystone individuals on collective behaviour scale to how long they remain within a group. Proceedings of the Royal Society B: Biological Sciences 282(1814): 20151766.

Ramos-Fernandez, G., A. J. King, J. C. Beehner, T. J. Bergman, M. C. Crofoot, A. Di Fiore, J. Lehmann, C. M. Schaffner, N. Snyder-Mackler \& K. Zuberbühler, 2018. Quantifying uncertainty due to fission-fusion dynamics as a component of social complexity. Proceedings of the Royal Society B: Biological Sciences 285(1879): 20180532.

Reader, S. M. \& K. N. Laland, 2002. Social intelligence, innovation, and enhanced brain size in primates. Proceedings of the National Academy of Sciences 99(7): 4436-4441.

Reddon, A. R., C. M. O'Connor, E. Nesjan, J. Cameron, J. K. Hellmann, I. Y. Ligocki, S. E. Marsh-Rollo, I. M. Hamilton, D. R. Wylie \& P. L. Hurd, 2017. Isotocin neuronal phenotypes differ among social systems in cichlid fishes. Royal Society Open Science 4(5): 170350.

Reddon, A. R., C. M. O'Connor, I. Y. Ligocki, J. K. Hellmann, S. E. Marsh-Rollo, I. M. Hamilton \& S. Balshine, 2016. No evidence for larger brains in cooperatively breeding cichlid fishes. Canadian Journal of Zoology 94(5): 373-378.

Richardson, J. L., M. C. Urban, D. I. Bolnick \& D. K. Skelly, 2014. Microgeographic adaptation and the spatial scale of evolution. Trends in Ecology \& Evolution 29(3): 165-176.

Rivas, L. R., 1964. A reinterpretation or the concepts "Sympatric" and "Allopatric" with proposal or the additional terms "Syntopic" and "Allotopic". Systematic Zoology 13(1): 42-43.

Roleira, A., G. A. Oliveira, J. S. Lopes \& R. F. Oliveira, 2017. Audience effects in territorial defense of male cichlid fish are associated with differential patterns of activation of the brain social decision-making network. Frontiers in Behavioral Neuroscience 11: 105.
Ronco, F., H. H. Büscher, A. Indermaur \& W. Salzburger, 2019. The taxonomic diversity of the cichlid fish fauna of ancient Lake Tanganyika, East Africa. Journal of Great Lakes Research 46: 1067-1078.

Rossiter, A., 1993. Studies on the biology of Neolamprologus multifasciatus. Ecological and limnological study on Lake Tanganyika and its adjacent regions VII. Kyoto: Kyoto University Press.

Rubenstein, D. R. \& P. Abbot, 2017. Comparative social evolution. Cambridge University Press, Cambridge.

Saeki, T., S. Sogawa, T. Hotta \& M. Kohda, 2018. Territorial fish distinguish familiar neighbours individually. Behaviour 155(4): 279-293.

Salonen, K., J. Sarvala, M. Järvinen, V. Langenberg, M. Nuottajärvi, K. Vuorio \& D. Chitamwebwa, 1999. Phytoplankton in Lake Tanganyika - vertical and horizontal distribution of in vivo fluorescence From Limnology to Fisheries: Lake Tanganyika and Other Large Lakes. Springer, Berlin: 89-103.

Salzburger, W., T. Mack, E. Verheyen \& A. Meyer, 2005. Out of Tanganyika: genesis, explosive speciation, key-innovations and phylogeography of the haplochromine cichlid fishes. BMC Evolutionary Biology 5(1): 17.

Sandel, A. A., J. A. Miller, J. C. Mitani, C. L. Nunn, S. K. Patterson \& L. Z. Garamszegi, 2016. Assessing sources of error in comparative analyses of primate behavior: Intraspecific variation in group size and the social brain hypothesis. Journal of Human Evolution 94: 126-133.

Sato, T., 1994. Active accumulation of spawning substrate: a determinant of extreme polygyny in a shell-brooding cichlid fish. Animal Behaviour 48(3): 669-678.

Sato, T. \& M. Gashagaza, 1997. Shell-brooding cichlid fishes of Lake Tanganyika: their habitats and mating systems. Fish Communities in Lake Tanganyika, pp. 219-240.

Sawaguchi, T., 1992. The size of the neocortex in relation to ecology and social structure in monkeys and apes. Folia Primatologica 58(3): 131-145.

Schädelin, F. C., S. Fischer \& R. H. Wagner, 2012. Reduction in predator defense in the presence of neighbors in a colonial fish. PLoS ONE 7(5): e35833.

Schädelin, F. C., W. F. van Dongen \& R. H. Wagner, 2015. Mate choice and genetic monogamy in a biparental, colonial fish. Behavioral Ecology 26(3): 782-788.

Schillaci, M. A., 2006. Sexual selection and the evolution of brain size in primates. PLoS ONE 1(1): e62.

Schradin, C. \& J. Lamprecht, 2002. Causes of female emigration in the group-living cichlid fish Neolamprologus multifasciatus. Ethology 108(3): 237-248.

Schuerch, R., S. Rothenberger \& D. Heg, 2010. The building-up of social relationships: behavioural types, social networks and cooperative breeding in a cichlid. Philosophical Transactions of the Royal Society B: Biological Sciences 365(1560): 4089-4098.

Schütz, D. \& M. Taborsky, 2000. Giant males or dwarf females: what determines the extreme sexual size dimorphism in Lamprologus callipterus? Journal of Fish Biology 57(5): 1254-1265.

Seehausen, O., 2006. African cichlid fish: a model system in adaptive radiation research. Proceedings of the Royal Society B: Biological Sciences 273(1597): 1987-1998. 
Sefc, K. M., 2011. Mating and parental care in Lake Tanganyika's cichlids. International Journal of Evolutionary Biology. https://doi.org/10.4061/2011/470875.

Shettleworth, S. J., 2009. Cognition, evolution, and behavior. Oxford University Press, Oxford.

Shultz, S. \& R. Dunbar, 2006. Both social and ecological factors predict ungulate brain size. Proceedings of the Royal Society B: Biological Sciences 273(1583): 207-215.

Shultz, S. \& R. I. Dunbar, 2007. The evolution of the social brain: anthropoid primates contrast with other vertebrates. Proceedings of the Royal Society B: Biological Sciences 274(1624): 2429-2436.

Shultz, S. \& R. I. Dunbar, 2010. Social bonds in birds are associated with brain size and contingent on the correlated evolution of life-history and increased parental investment. Biological Journal of the Linnean Society 100(1): 111-123.

Shumway, C. A., 2008. Habitat complexity, brain, and behavior. Brain, Behavior and Evolution 72(2): 123-134.

Shumway, C. A., 2010. The evolution of complex brains and behaviors in African cichlid fishes. Current zoology 56(1): 144-156.

Shumway, C. A., H. A. Hofmann \& A. P. Dobberfuhl, 2007. Quantifying habitat complexity in aquatic ecosystems. Freshwater Biology 52(6): 1065-1076.

Simões, J. M., M. C. Teles, R. F. Oliveira, A. Van der Linden \& M. Verhoye, 2012. A three-dimensional stereotaxic MRI brain atlas of the cichlid fish Oreochromis mossambicus. PLoS ONE 7(9): e44086.

Slotow, R. \& E. Paxinos, 1997. Intraspecific competition influences food return-predation risk trade-off by Whitecrowned Sparrows. The Condor 99(3): 642-650.

Smith, J. M., 1977. Parental investment: A prospective analysis. Animal Behaviour. 25: 1-9.

Smuts, B. B., D. L. Cheney, R. M. Seyfarth \& R. W. Wrangham, 2008. Primate societies. University of Chicago Press, Chicago.

Soares, M. C., G. I. André \& J. R. Paula, 2015. Preliminary notes on brain weight variation across labrid fish species with different levels of cooperative behaviour. Current Zoology 61(2): 274-280.

Sopinka, N., J. Fitzpatrick, J. Desjardins, K. Stiver, S. MarshRollo \& S. Balshine, 2009. Liver size reveals social status in the African cichlid Neolamprologus pulcher. Journal of Fish Biology 75(1): 1-16.

Stowers, J. R., M. Hofbauer, R. Bastien, J. Griessner, P. Higgins, S. Farooqui, R. M. Fischer, K. Nowikovsky, W. Haubensak \& I. D. Couzin, 2017. Virtual reality for freely moving animals. Nature Methods 14(10): 995.

Sturmbauer, C., W. Salzburger, N. Duftner, R. Schelly \& S. Koblmüller, 2010. Evolutionary history of the Lake Tanganyika cichlid tribe Lamprologini (Teleostei: Perciformes) derived from mitochondrial and nuclear DNA data. Molecular Phylogenetics and Evolution 57(1): 266-284.

Sturmbauer, C., E. Verheyen \& A. Meyer, 1994. Mitochondrial phylogeny of the Lamprologini, the major substrate spawning lineage of cichild fishes from Lake Tanganyika in eastern Africa. Molecular Biology and Evolution 11(4): 691-703.
Sunobe, T. \& H. Munehara, 2003. Mating system and kin relationship between adults and young in the shell-brooding cichlid fish Neolamprologus meeli in Lake Tanganyika. Journal of Ethology 21(2): 87-92.

Sylvester, J. B., C. A. Rich, Y.-H. E. Loh, M. J. van Staaden, G. J. Fraser \& J. T. Streelman, 2010. Brain diversity evolves via differences in patterning. Proceedings of the National Academy of Sciences 107(21): 9718-9723.

Szathmáry, E. \& J. M. Smith, 1995. The major evolutionary transitions. Nature 374(6519): 227-232.

Taborsky, B., C. Arnold, J. Junker \& A. Tschopp, 2012. The early social environment affects social competence in a cooperative breeder. Animal Behaviour 83(4): 1067-1074.

Taborsky, B. \& R. F. Oliveira, 2012. Social competence: an evolutionary approach. Trends in Ecology \& Evolution 27(12): 679-688.

Taborsky, B., L. Tschirren, C. Meunier \& N. Aubin-Horth, 2013. Stable reprogramming of brain transcription profiles by the early social environment in a cooperatively breeding fish. Proceedings of the Royal Society B: Biological Sciences 280(1753): 20122605.

Taborsky, M. \& D. Limberger, 1981. Helpers in fish. Behavioral Ecology and Sociobiology 8(2): 143-145.

Taborsky, M., 1994. Sneakers, satellites, and helpers: parasitic and cooperative behavior in fish reproduction. Advances in the Study of Behavior 23(1): e100.

Taborsky, M., L. Brouwer, D. Heg \& Z. Bachar, 2005. Large group size yields group stability in the cooperatively breeding cichlid Neolamprologus pulcher. Behaviour 142(11-12): 1615-1641.

Taborsky, M., S. Koblmüller, K. M. Sefc, M. McGee, M. Kohda, S. Awata, M. Hori, et al., 2019. Insufficient data render comparative analyses of the evolution of cooperative breeding mere speculation: A reply to Dey et al., Ethology 125(11): 851-854.

Taborsky, M. \& M. Wong, 2017. 12 Sociality in Fishes. Comparative Social Evolution 354: 11.

Takahashi, T., 2004. Morphological and genetic distinctness of rock and shell-bed dwelling Telmatochromis (Teleostei, Cichlidae) in the south of Lake Tanganyika suggest the existence of two species. Journal of Fish Biology 65(2): 419-435.

Takahashi, T., 2019. Colour variation of a shell-brooding cichlid fish from Lake Tanganyika. Hydrobiologia 832(1): 193-200.

Takahashi, T. \& S. Koblmüller, 2011. The adaptive radiation of cichlid fish in Lake Tanganyika: a morphological perspective. International Journal of Evolutionary Biology. https://doi.org/10.4061/2011/620754.

Takahashi, T. \& K. Ota, 2016. Body size evolution of a shellbrooding cichlid fish from Lake Tanganyika. Journal of Evolutionary Biology 29(12): 2373-2382.

Takahashi, T., K. Ota, M. Kohda \& M. Hori, 2012. Some evidence for different ecological pressures that constrain male and female body size. Hydrobiologia 684(1): 35-44.

Takahashi, T., K. Watanabe, H. Munehara, L. Rüber \& M. Hori, 2009. Evidence for divergent natural selection of a Lake Tanganyika cichlid inferred from repeated radiations in body size. Molecular Ecology 18(14): 3110-3119.

Tanaka, H., J. G. Frommen, S. Koblmüller, K. M. Sefc, M. McGee, M. Kohda, S. Awata, M. Hori \& M. Taborsky, 
2018. Evolutionary transitions to cooperative societies in fishes revisited. Ethology 124(11): 777-789.

Taves, M. D., J. K. Desjardins, S. Mishra \& S. Balshine, 2009. Androgens and dominance: sex-specific patterns in a highly social fish (Neolamprologus pulcher). General and Comparative Endocrinology 161(2): 202-207.

Teles, M. C., O. Almeida, J. S. Lopes \& R. F. Oliveira, 2015. Social interactions elicit rapid shifts in functional connectivity in the social decision-making network of zebrafish. Proceedings of the Royal Society B: Biological Sciences 282(1816): 20151099.

Teles, M. C., S. D. Cardoso \& R. F. Oliveira, 2016. Social plasticity relies on different neuroplasticity mechanisms across the brain social decision-making network in zebrafish. Frontiers in Behavioral Neuroscience 10: 16.

Thornton, A. \& K. McAuliffe, 2015. Cognitive consequences of cooperative breeding? A critical appraisal. Journal of Zoology 295(1): 12-22.

Triki, Z., E. Levorato, W. McNeely, J. Marshall \& R. Bshary, 2019. Population densities predict forebrain size variation in the cleaner fish Labroides dimidiatus. Proceedings of the Royal Society B 286(1915): 20192108.

Trillmich, F., G. Brandtmann \& M. Scandura, 1999. Femalefemale conflict in the harem of a snail cichlid (Lamprologus ocellatus): behavioural interactions and fitness consequences. Behaviour 136(9): 1123-1144.

Tsuboi, M., A. Gonzalez-Voyer \& N. Kolm, 2014. Phenotypic integration of brain size and head morphology in Lake Tanganyika cichlids. BMC Evolutionary Biology 14(1): $1-10$.

Tsuboi, M., A. Husby, A. Kotrschal, A. Hayward, S. D. Buechel, J. Zidar, H. Løvlie \& N. Kolm, 2015. Comparative support for the expensive tissue hypothesis: big brains are correlated with smaller gut and greater parental investment in Lake Tanganyika cichlids. Evolution 69(1): 190-200.

van der Bijl, W. \& N. Kolm, 2016. Why direct effects of predation complicate the social brain hypothesis: and how incorporation of explicit proximate behavioral mechanisms might help. BioEssays 38(6): 568-577.

van Doorn, G. S. \& M. Taborsky, 2012. The evolution of generalized reciprocity on social interaction networks. Evolution: International Journal of Organic Evolution 66(3): 651-664.

van Staaden, M. J., P. Huber, L. S. Kaufman \& K. F. Liem, 1994. brain and body size, general patterns, and evolutionary trends. Zoology 98(95): 165-178.
Varela, S. A., M. C. Teles \& R. F. Oliveira, 2020. The correlated evolution of social competence and social cognition. Functional Ecology 34(2): 332-343.

Weisbecker, V., S. Blomberg, A. W. Goldizen, M. Brown \& D. Fisher, 2015. The evolution of relative brain size in marsupials is energetically constrained but not driven by behavioral complexity. Brain, Behavior and Evolution 85(2): 125-135.

Weitekamp, C. A. \& H. A. Hofmann, 2017. Neuromolecular correlates of cooperation and conflict during territory defense in a cichlid fish. Hormones and Behavior 89: 145-156.

Wey, T., D. T. Blumstein, W. Shen \& F. Jordán, 2008. Social network analysis of animal behaviour: a promising tool for the study of sociality. Animal Behaviour 75(2): 333-344.

Whiten, A. \& R. W. Byrne, 1988. Tactical deception in primates. Behavioral and Brain Sciences 11(2): 233-244.

Wickler, W., 1962. Zur Stammesgeschichte funktionell korrelierter Organ-und Verhaltensmerkmale: Ei-Attrappen und Maulbrüten bei afrikanischen Cichliden. Zeitschrift für Tierpsychologie 19(2): 129-164.

Widmer, L., E. Heule, M. Colombo, A. Rueegg, A. Indermaur, F. Ronco \& W. Salzburger, 2019. Point-combination transect (PCT): incorporation of small underwater cameras to study fish communities. Methods in Ecology and Evolution 10(6): 891-901.

Wilson, E. O., 2000. Sociobiology: the new synthesis. Harvard University Press, Cambridge.

Winkelmann, K., M. J. Genner, T. Takahashi \& L. Rüber, 2014. Competition-driven speciation in cichlid fish. Nature communications 5(1): 1-8.

Wismer, S., A. I. Pinto, A. L. Vail, A. S. Grutter \& R. Bshary, 2014. Variation in cleaner wrasse cooperation and cognition: influence of the developmental environment? Ethology $120(6)$ : 519-531.

Wrona, F. J. \& R. J. Dixon, 1991. Group size and predation risk: a field analysis of encounter and dilution effects. The American Naturalist 137(2): 186-201.

Yuma, M., 1994. Food habits and foraging behaviour of benthivorous cichlid fishes in Lake Tanganyika. Environmental Biology of Fishes 39(2): 173-182.

Publisher's Note Springer Nature remains neutral with regard to jurisdictional claims in published maps and institutional affiliations. 